\title{
Extent and Mechanism of Sealing in Transected Giant Axons of Squid and Earthworms
}

\author{
Todd L. Krause, ${ }^{1,2}$ Harvey M. Fishman, ${ }^{4}$ Martis L. Ballinger, ${ }^{1}$ and George D. Bittner ${ }^{1,2,3}$ \\ 'Department of Zoology, ${ }^{2}$ Institute for Neuroscience, and ${ }^{3}$ College of Pharmacy, University of Texas, Austin, Texas 78712 \\ and ${ }^{4}$ Department of Physiology and Biophysics, University of Texas Medical Branch, Galveston, Texas 77555-0641
}

\begin{abstract}
Transected axons are often assumed to seal at their cut ends by the formation of continuous membrane barriers that allow for the restoration of function in the axonal stumps. We have used several electrophysiological measures (membrane potential, input resistance, injury current density) and several morphological measures (phase-contrast, video-enhanced differential interference contrast, light, and electron microscopies) of living and fixed material to assess the extent and mechanism of sealing within hours after transecting giant axons of squid (Loligo pealei and Sepioteuthis lessoniana) and earthworms (Lumbricus terrestris). Our electrophysiological data suggest that the proximal and distal ends of transected squid giant axons do not completely seal within $2.5 \mathrm{hr}$ in physiological saline. In contrast, the same set of measures suggest that proximal and distal onds of transected earthworm giant axons seal within $1 \mathrm{hr}$ in physiological saline. Our morphological data show that the cut ends of both squid and earthworm axons constrict, but that a 2070- $\mu$ m-diameter opening always remains at the cut end that is filled with vesicles. Axonal transection induces the formation of vesicles that are observed in the axoplasm within minutes in standard salines and that rapidly migrate to the cut ends. These injury-induced vesicles are loosely packed near the cut ends of squid giant axons, which do not functionally seal within $2.5 \mathrm{hr}$ of transection. In contrast, vesicles formed a tightly packed plug at the cut ends of earthworm medial giant axons, which do functionally seal within $1 \mathrm{hr}$ of transection in physiological saline. Since we detect no single continuous membrane that spans the cut end, sealing does not appear to occur by the fusion of constricted axolemmal membrane or the formation of a membranous partition at the cut end. Rather, our data are consistent with the hypothesis that a tightly packed vesicular plug is responsible for sealing of earthworm giant axons.
\end{abstract}

[Key words: axotomy, injury, injury current, injury-induced vesiculation, repair, sealing assessment, sealing mechanisms, vesicular plug]

\footnotetext{
Received Oct. 7, 1993; revised Mar. 16, 1994; accepted Apr. 27, 1994.

We thank the Zeiss, Central Microscope, and National Vibrating Probe Facilities at the Marine Biological Laboratory (Woods Hole, MA) for use of their equipment. We thank Dr. Peter Smith, Mr. Alan Shipley, Mr. Philip Presley, Mr. Louis Kerr, and Dr. Janis Metuzals for discussions and technical assistance. We also thank the Marine Biomedical Institute in Galveston for supplying cephalopods (NIH RR01024). This work was supported in part by NIH Grant NS31256 and ONR Grant N00014-90-J-1 137 to H.M.F., an NIAAA fellowship to T.L.K., and an ATP grant to G.D.B.

Correspondence should be addressed to Dr. Harvey M. Fishman at the above address.

Copyright (c) 1994 Society for Neuroscience $0270-6474 / 94 / 146638-14 \$ 05.00 / 0$
}

After transection, the proximal portion of a severed axon may survive and even regenerate; the distal portion degenerates within hours to days in mammals and birds, but often survives for weeks to years in lower vertebrates and invertebrates (see Bittner, 1991, for a review). To survive, the proximal or distal portion of a severed axon must form a barrier (seal) at the transection site to prevent toxic changes in the internal concentrations of ions or macromolecules. Such a seal should be discernible both functionally and morphologically.

This article reports data from a set of electrophysiological measures [membrane potential $\left(v_{m}\right)$, input resistance $\left(R_{\mathrm{in}}\right)$, and injury-induced current density $\left.\left(I_{\text {in }}\right)\right]$ and morphological measures of living [phase-contrast microscopy, or video-enhanced differential interference contrast microscopy (VE-DIC)] and fixed [light microscopy, and transmission electron microscopy (TEM)] tissue. Data from each of these complementary measures are used to evaluate the extent and mechanism of sealing following transection of unmyelinated giant axons (GAs) of squid (Loligo pealei and Sepioteuthis lessoniana) and the invertebrate-myelinated medial giant axon (MGA) of the earthworm (Lumbricus terrestris). The use of giant axons allowed us to make these measures on single, identifiable axons whose characteristics are repeatable from preparation to preparation. These two particular giant axons were chosen because they have been extensively characterized both electrophysiologically and morphologically. Furthermore, although axonal regeneration has not been well characterized in squid GAs (Sereni and Young, 1932), there are numerous reports (Bullock and Horridge, 1965; Birse and Bittner, 1981; Lyckman et al., 1992) of earthworm MGA regeneration (suggesting their ability to seal).

Our electrophysiological data show that the squid GA does not completely seal within $2.5 \mathrm{hr}$. In contrast, analyses using these measures show that the earthworm MGA does seal within $1 \mathrm{hr}$. These electrophysiological data correlate with our morphological observations that the axolemma at the cut ends of squid GAs constrict by $80-95 \%$ and that large vesicles are loosely packed in the vicinity of the cut end within $2.5 \mathrm{hr}$ of axonal transection in standard saline. In contrast, the axolemma at the cut ends of earthworm MGAs constricts about $50-70 \%$ and large vesicles are densely packed at the cut end within 1 hour of axonal transection in standard saline. Our electrophysiological and morphological data are not consistent with the conventional notion that sealing occurs by constriction and fusion of axolemmal membranes at the cut end. Further, our data are not consistent with the view that sealing occurs by the appearance of a single membrane that spans the opening at or near the cut axonal end. However, all our electrophysiological and morphological data are consistent with the hypothesis that rapid axonal sealing 
is mediated by the formation of a vesicular plug at the cut end of earthworm MGAs.

\section{Materials and Methods}

\section{Experimental preparations}

Squid. Two species of squid were used so we could conduct experiments year round: one from the Marine Biological Laboratory, Woods Hole, MA (Ioligo pealei) and the other from the Marine Biomedical Institute, Galveston, TX (Sepioteuthis lessoniana) (GAs from these species have similar electrophysiological and morphological characteristics; Lee et al., 1994). Squid were decapitated and 3-5 cm lengths of GAs were removed from the hindmost stellar nerve after ligating the ends with cotton thread or \#7.0 silk suture (Gilbert et al., 1990). In most experiments as noted, the anucleated GAs were placed in artificial sea water (standard ASW) composed of $430 \mathrm{~mm} \mathrm{NaCl}, 10 \mathrm{~mm} \mathrm{KCl}, 10 \mathrm{~mm} \mathrm{CaCl}_{2}$, $50 \mathrm{~mm} \mathrm{MgCl}_{2}$, and $5 \mathrm{~mm}$ Tris $\mathrm{Cl}$ (pH 7.4, $980 \mathrm{mOsm}$ ). In other cases, GAs were placed in isosmotic divalent cation-free saline (divalent cation-free ASW) to which $180 \mathrm{~mm}$ tetramethylammonium chloride and

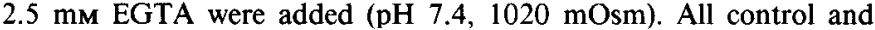
experimental observations were made at room temperature $\left(20-24^{\circ} \mathrm{C}\right)$. Although GAs from both species of squid exhibited similar electrophysiological and morphological changes following transection, we have not combined data from the two species. Rather, data are presented from only one species, as indicated. No differences were noted in any measure for the proximal versus distal cut ends of scvered GAs. Data from both proximal and distal ends were combined for each species of squid.

GAs ranged from 300 to $600 \mu \mathrm{m}$ in diameter. GAs are surrounded by a sheath $5-10 \mu \mathrm{m}$ wide. The innermost portion of the sheath consists of an adaxonal layer of glial cytoplasm somewhat analogous to that which surrounds unmyelinated vertebrate axons (Villegas and Villegas, 1984; Brown and Lasek, 1990). The remainder of the sheath consists primarily of connective tissue.

Earthworm. Earthworms (Lumbricus terrestris) were obtained from local bait shops and maintained in a large dirt-filled container where they were fed regularly. Sexually mature earthworms were anesthetized in $0.2 \%(\mathrm{w} / \mathrm{w})$ chloretone, rinsed, pinned on a paraffin dish, and opened by a longitudinal midventral incision. The ventral nerve cord containing the MGA was then dissected frec of the animal. In most experiments as noted, the MGA was maintained in physiological earthworm saline (standard EWS) containing $40.1 \mathrm{~mm} \mathrm{NaCl}, 10 \mathrm{mM} \mathrm{Na} \mathrm{SO}_{2}, 25 \mathrm{~mm} \mathrm{Na}$ acetate, $0.5 \mathrm{mM} \mathrm{K}_{2} \mathrm{SO}_{4}, 3.0 \mathrm{~mm} \mathrm{CaCl}, 0.5 \mathrm{mM} \mathrm{MgCl}_{2}, 1.25 \mathrm{~mm}$ Tris, and 1.4 mM HEPES (pH 7.4, $181 \mathrm{mOsm}$ ). In other cases, the MGA was placed in an isosmotic divalent cation-free EWS to which $7 \mathrm{~mm} \mathrm{NaCl}$ and $2.5 \mathrm{~mm}$ EGTA were added (pH 7.4, $195 \mathrm{mOsm}$ ). All control and experimental observations were made at room temperature $\left(20-24^{\circ} \mathrm{C}\right)$. No differences in any measure were noted for the proximal versus distal cut end of severed MGAs. Data from both proximal and distal ends were combined.

MGAs ranged from 70 to $110 \mu \mathrm{m}$ in diameter. MGAs are surrounded by a membranous glial sheath 3-9 $\mu \mathrm{m}$ in width composed of $50-200$ lamellae (Coggeshall, 1965; Günther, 1976). This glial sheath is analogous to vertebrate myelin in its morphology and function. Each MGA extends for one body segment (1-2 $\mathrm{mm}$ in length) and is electrotonically connected to MGAs in adjacent segments by septa containing gap junctions (Brink and Barr, 1977; Verselis and Brink, 1984).

Prior to transection, intact squid GAs and earthworm MGAs were examined in standard or divalent cation-free salines. Damaged axons with intracellular vesicles and/or cytoplasmic opacities (viewed at $\leq 200 \times$ ) were discarded.

\section{Electrophysiological analyses}

Intracellular measures $\left(\mathrm{v}_{\mathrm{m}}\right.$ and $\left.\mathrm{R}_{\text {in }}\right)$. GAs and MGAs were intracellularly impaled with two glass microelectrodes $(4-10 \mathrm{M} \Omega$ ) filled with $3 \mathrm{M} \mathrm{KCl}$. $v_{m}$ and $R_{\text {in }}$ were determined using one microelectrode to measure the potential and the other to apply current a short distance away $(40-80$ $\mu \mathrm{m})$. To enhance the localization of applied current, we covered the tied ends of dissected GAs in a mixture of petroleum jelly and mineral oil. [This procedure was unnecessary in MGAs because their septal membranes enhanced current localization.] Subthreshold rectangular current pulses of 20-50 nA and 5-6 msec duration were applied to obtain $R_{\mathrm{in}}$, where $R_{\text {in }}$ equaled the magnitude of the steady state potential change divided by the magnitude of the applied current.
Measures of $v_{m}$ and $R_{\text {in }}$ were made before and after transection of squid GAs and earthworm MGAs. To avoid damage to the axolemma at sites other than the lesion site (Yawo and Kuno, 1985), we removed the microelectrodes before transecting the axon in its middle with scissors or a microknife made from a shard of a Gillette Blue Blade. Axons were reimpaled a short distance $(60-400 \mu \mathrm{m})$ from the cut axonal end a brief time ( $\leq 3 \mathrm{~min}$ ) after transection. Changes in $v_{m}$ and $R_{\text {in }}$ during sealing of GAs and MGAs were continuously monitored for up to $4 \mathrm{hr}$. We assessed the effect of multiple microelectrode impalements in intact GAs and MGAs and found that they could be impaled repeatedly without a noticeable decrease in resting potential. To ensure that there was no membrane barrier (septa) between the microelectrodes and the site of transection in MGAs, septal location was sometimes confirmed in intact or severed axons by injection of Lucifer yellow dye (Krause and Bittner, 1990).

Extracellular measure of $\mathrm{I}_{i n j}$. The "vibrating probe" technique (Jaffe and Nuccitelli, 1974; Scheffey, 1988) is a sensitive way to monitor current density by determining the voltage between two points in an extracellular medium of known resistivity. An intact (control) axon usually has small local currents. When an axon is transected, ions move down their concentration gradients to create a large net inward $I_{\mathrm{inj}}$ at the cut end of the axon and a net outward current that decays with distance from the cut end along the axon (Borgens et al., 1980, 1989). As an axon recovers from transection, the inward $I_{\text {inj }}$ at the cut end should decline to control values once a complete seal is formed. The $I_{\text {inj }}$ measured extracellularly with a vibrating probe is independent of changes in axonal cable properties, which complicate the evaluation of more common electrophysiological measures (i.e., $v_{m}$ and $R_{\mathrm{in}}$ ) of sealing (see Discussion).

$I_{\text {inj }}$ was measured at the National Vibrating Probe Facility (Marine Biological Laboratory, Woods Hole, MA) using an extracellular twodimensional voltage-sensitive vibrating probe (Scheffey, 1988; Smith and Shipley, 1990). Dissected axons were pinned out in a Sylgard-coated dish containing standard or divalent cation-free salines. Current densities were measured from intact (control) GAs and MGAs in standard salines by placing the tip of the probe a fixed distance from the surface of the axons at various points along their lengths. After transecting an axon, the probe was again placed the fixed distance from the surface of the axon at various points along its length and its cut end. This fixed distance from the axonal surface or the cut end was measured for each preparation and maintained throughout the entire experiment as later confirmed by review of continuous video records made of all preparations. In some experiments the contribution of current from surrounding tissues was minimized by aligning the probe with the long axis of the axon and monitoring the current density only along this axis. For these experiments, we used $I_{\max }$ as a measure of $I_{\mathrm{ini}}$. $I_{\max }$ was defined as the maximum net current density parallel to the longitudinal axis of the axon measured at the fixed distance from the cut end. In all experiments, electrical drift was eliminated by moving the probe to a reference position far from the cut end to obtain zero current density prior to each sample of the $I_{\mathrm{inj}}$. The probe was then returned to the point of maximal $I_{\mathrm{inj}}$ at the fixed distance from the cut end.

\section{Morphological analyses}

Morphology of living axons. Axons were viewed in vitro with phasecontrast and VE-DIC microscopy using a Zeiss Axiophot or Axiovert35 light microscope equipped with differential interference contrast (DIC) optics and $20 \times$ or $40 \times$ LD Achroplan objectives (numerical aperture, 0.40 and 0.60 , respectively), Newvicon video tube, Hamamatsu C2400 camera and Argus 10 image processor. To assess morphological changes associated with axonal transection, we viewed GAs and MGAs before and after ( $\leq 1-180 \mathrm{~min})$ axonal transection in standard or divalent cation-free salines. When viewed at lower magnifications $(\leq 200 \times)$, the axons were placed in open air chambers to facilitate axonal transection and solution changes. When viewed at higher magnifications (200$1000 \times$ ), the preparation was placed in a solution-filled trough formed with silicone grease on a glass slide, and sealed with a cover slip.

Morphology of fuxed axons. Intact and severed squid GAs were fixed for 2-2.5 hr in a phosphate buffer (22 $\mathrm{mM} \mathrm{NaH}_{2} \mathrm{PO}_{4}, 78 \mathrm{mM} \mathrm{Na}_{2} \mathrm{HPO}_{4}$, $625 \mathrm{~mm}$ sucrose, $0.1 \mathrm{mM}$ EGTA; pH 7.4, 990 mOsm) containing $2 \%$ glutaraldehyde. GAs were postfixed for $2 \mathrm{hr}$ in $2 \% \mathrm{OsO}_{4}$ in phosphate buffer $(0.1 \mathrm{M})$, dehydrated in an ethanol series, cleared with propylene oxide, and embedded in Epon plastic (Gilbert et al., 1990). Intact and severed earthworm MGAs were fixed for $2 \mathrm{hr}$ in a phosphate buffer (22 mм NaH $\mathrm{PO}_{4}, 78 \mathrm{~mm} \mathrm{Na}_{2} \mathrm{HPO}_{4} ; \mathrm{pH} 7.4,210 \mathrm{mOsm}$ ) containing $1.25 \%$ 

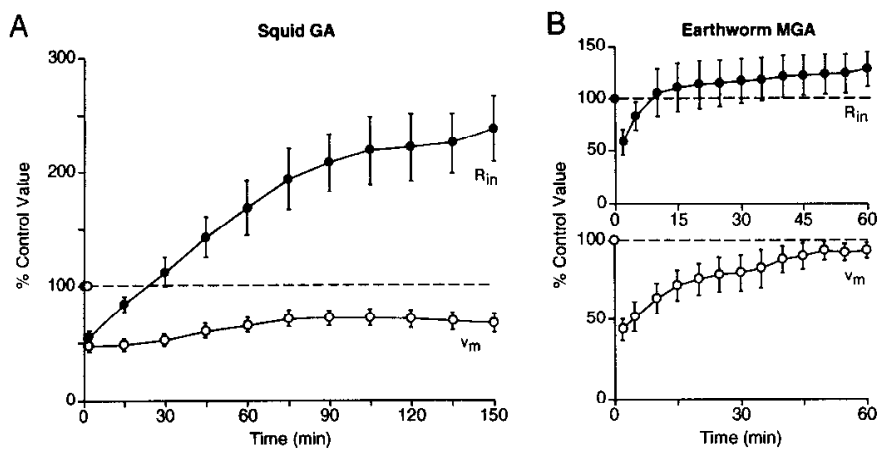

Figure 1. Variation of $v_{m}$ (open circles) and $R_{\text {in }}$ (solid circles) with time after transection of squid GAs and earthworm MGAs in standard salines plotted as a percentage of precut $(0 \mathrm{~min})$ values. $v_{m}$ and $R_{\mathrm{in}}$ were measured with two microelectrodes placed intracellularly a short distance $(\leq 400 \mu \mathrm{m})$ from the site of axonal transection. Dashed lines indicate precut values (set to $100 \%)$, and error bars represent \pm 1 SEM $(68 \%$ confidence interval). $A$, Squid (Sepioteuthis lessoniana) GAs $(n=5)$ transected at time $0 \mathrm{~min}$ and monitored for $150 \mathrm{~min}$ following transection. $v_{m}$ and $R_{\text {in }}$ before $(0 \mathrm{~min})$ axonal transection were $-56.8 \pm 2.1$ $\mathrm{mV}$ and $3.5 \pm 0.2 \mathrm{k} \Omega$, respectively. Immediately after transection, $v_{m}$ and $R_{\text {in }}$ were about $50 \%$ of precut values. At 150 min following transection, $v_{m}$ and $R_{\text {in }}$ were $66 \%$ and $234 \%$ of precut values, respectively. $B$, Earthworm MGAs $(n=4)$ transected at time 0 min and monitored for $60 \mathrm{~min}$ following transection. $v_{m}$ and $R_{\text {in }}$ before $(0 \mathrm{~min})$ axonal transection were $-70.8 \pm 2.2 \mathrm{mV}$ and $279 \pm 68.1 \mathrm{k} \Omega$, respectively. Immediately after transection, $v_{m}$ and $R_{\text {in }}$ were about $50 \%$ of precut values; $v_{m}$ and $R_{\mathrm{in}}$ at $60 \mathrm{~min}$ following transection, were $94 \%$ and $122 \%$ of precut values, respectively. Following axonal transection, $v_{m}$ did not recover in squid GAs, but did recover in earthworm MGAs. $R_{\text {in }}$ eventually exceeded precut values in both GAs and MGAs.

glutaraldehyde. MGAs were postfixed for $1.5 \mathrm{hr}$ in $2 \% \mathrm{OsO}_{4}$ in $0.1 \mathrm{M}$ phosphate buffer, dehydrated in an ethanol series, and embedded in Spurrs' plastic. GAs and MGAs were cut longitudinally in thick and thin sections for viewing with light and electron microscopes, respectively. For low-magnification $(10-500 \times)$ analyses, thick sections were stained with Richardson's stain and viewed with a Zeiss Axiophot microscope. For high-magnification $(2000-50,000 \times)$ analyses, thin sections were collected on single-hole Formvar-coated grids, stained with lead citrate, and viewed with a Siemens (IA) transmission electron microscope at $60 \mathrm{kV}$.

\section{Results}

Changes in $\mathrm{v}_{\mathrm{m}}$ and $\mathbf{R}_{\text {in }}$ following transection of $G A$ s and $M G A s$

Prior to transection, $v_{m}$ and $R_{\text {in }}$ were measured in intact squid GAs $(n=5)$ and intact earthworm MGAs $(n=4)$ maintained in standard salines. We confirmed values previously published for $v_{m}$ and $R_{\text {in }}$ as follows: for the squid GA, $v_{m}=-56.8 \pm 2.1$ $\mathrm{mV}\left[\right.$ mean $\pm 1 \mathrm{SEM}(68 \%$ confidence interval) $]$ and $R_{\mathrm{in}}=3.5$ $\pm 0.2 \mathrm{k} \Omega$; for the earthworm MGA, $v_{m}=-70.8 \pm 2.2 \mathrm{mV}$ and $R_{\text {in }}=279 \pm 68.1 \mathrm{k} \Omega$.

Following transection, $v_{m}$ measured within 3 min dropped to $46 \%(-26.4 \pm 3.1 \mathrm{mV})$ of control valucs in intact squid GAs (Fig. $1 A)$ and to $44 \%(-31.3 \pm 5.3 \mathrm{mV})$ in earthworm MGAs (Fig. $1 B$ ). For about $90 \mathrm{~min}, v_{m}$ in GAs slowly recovered to $71 \%$ of control values measured in intact axons. This slow increase was followed by a slow decline in $v_{m}$ (Fig. $1 A$ ), which probably reflected degenerative changes in squid GAs ( $v_{m}$ in intact squid GAs did not change for many hours). In contrast, $v_{m}$ in earthworm MGAs $(n=4)$ recovered to $90-100 \%(-66.7 \pm 5.2 \mathrm{mV})$ of control values in intact axons within 60 min after transection and remained stable for hours thereafter (Fig. $1 B$ ). That is, the $v_{m}$ of MGAs 60 minutes after axonal transection was not significantly different $\left(P>0.05\right.$, Student's $t$ test) from the $v_{m}$ of these axons before transection.

Following transection of squid GAs, $R_{\text {in }}$ (Fig. $1 A$ ) recovered over a $2.5 \mathrm{hr}$ period, increasing from $1.9 \pm 0.3 \mathrm{k} \Omega$ at $\leq 3 \mathrm{~min}$ to $8.2 \pm 0.9 \mathrm{k} \Omega$. Following transection of earthworm MGAs, $R_{\text {in }}$ (Fig. $1 B$ ) recovered within $60 \mathrm{~min}$, incrcasing from $149.8 \pm$ $27.9 \mathrm{k} \Omega$ at $\leq 3 \mathrm{~min}$ to $341.3 \pm 68.5 \mathrm{k} \Omega$. On average, posttransection values for $R_{\text {in }}$ were $234 \%$ of control values in intact GAs and $122 \%$ of control values in intact MGAs.

In several other experiments (not shown), we found that $v_{m}$ and axonal resistance did not recover when squid GAs or earthworm MGAs were transected in divalent cation-free salines. These data are consistent with reports that extracellular calcium is necessary for repair of damaged cell membranes and hence for recovery of $v_{m}$ and $R_{\text {in }}$ (Deleze, 1970; Nishiye, 1977; Yawo and Kuno, 1983, 1985).

\section{Changes in injury current following transection of $G$ As and $M G A s$}

A two-dimensional vibrating probe enables measurement of the direction and magnitude of current density at discrete locations in cxtraccllular solution (Jaffe and Nuccitelli, 1974). The direction and magnitude of the current density vector at each location in the solution can be represented by the direction and length of an arrow (Fig. 2). Each arrow originates from the point in the solution at which the measurement was made (i.e., the location of the tip of the vibrating probe).

Control current density vectors were determined by placing the tip of the vibrating probe a fixed distance from the axon at various locations along the length of intact GAs and MGAs dissected free of surrounding tissue (Fig. $2 A, B$ ). Intact squid GAs $(n=3)$ had an even distribution of outward control current density vectors along their lengths (Fig. $2 A$ ). Intact earthworm MGAs $(n=7)$ dissected free of surrounding tissue had relatively

Figure 2. Distribution of current density along the length, and at the cut end of control and transected squid GAs (left column) and earthworm MGAs (right column) in standard saline. Arrows indicate the magnitude (arrow length) of current density and the direction of current flow. GAs of Loligo peale $i$ were used in these experiments. $A$ and $B$, Distribution of current along undamaged (control) axons. In $A$, the control squid $\mathrm{GA}$ had current evenly distributed along its length. In $B$, the control earthworm MGA partially isolated from the ventral nerve cord (*) had an uneven distribution of current with the largest current entering the ventral nerve cord. $C$ and $D$, Distribution of current along the length and at the cut end of transected axons. In $C$, the squid GA 10 min after transection had a large, localized inward current at the cut axonal end. In $D$, the earthworm MGA $30 \mathrm{~min}$ after transection had large inward currents localized at the cut axonal end and at the ventral nerve cord. $E$ and $F$, Distribution of current along the length and at the cut end of squid GAs $2.5 \mathrm{hr}$ after transection and earthworm MGAs $1 \mathrm{hr}$ after transection. In $E$, the squid GA $2.5 \mathrm{hr}$ after transection had a persistent large inward current localized at the cut axonal end. In $F$, the earthworm MGA $1 \mathrm{hr}$ after transection had inward currents of equal, small magnitudes evenly distributed along the axon and at the cut axonal end. Note that there was still a large current localized at the cut end of squid GAs $2.5 \mathrm{hr}$ after transection. In contrast, the current induced by injury in earthworm MGAs decreased to the level found in control axons by $1 \mathrm{hr}$ after transection. Scale bar (given $E$ ): $A, 20 \mu \mathrm{A} / \mathrm{cm}^{2}, 360 \mu \mathrm{m} ; B, 10 \mu \mathrm{A} / \mathrm{cm}^{2}, 80 \mu \mathrm{m} ; C, 400 \mu \mathrm{A} / \mathrm{cm}^{2}, 360 \mu \mathrm{m}, D$, $10 \mu \mathrm{A} / \mathrm{cm}^{2}, 80 \mu \mathrm{m} ; E, 40 \mu \mathrm{A} / \mathrm{cm}^{2}, 360 \mu \mathrm{m} ;$ and $F, 10 \mu \mathrm{A} / \mathrm{cm}^{2}, 80 \mu \mathrm{m}$. 


\section{Squid GA}
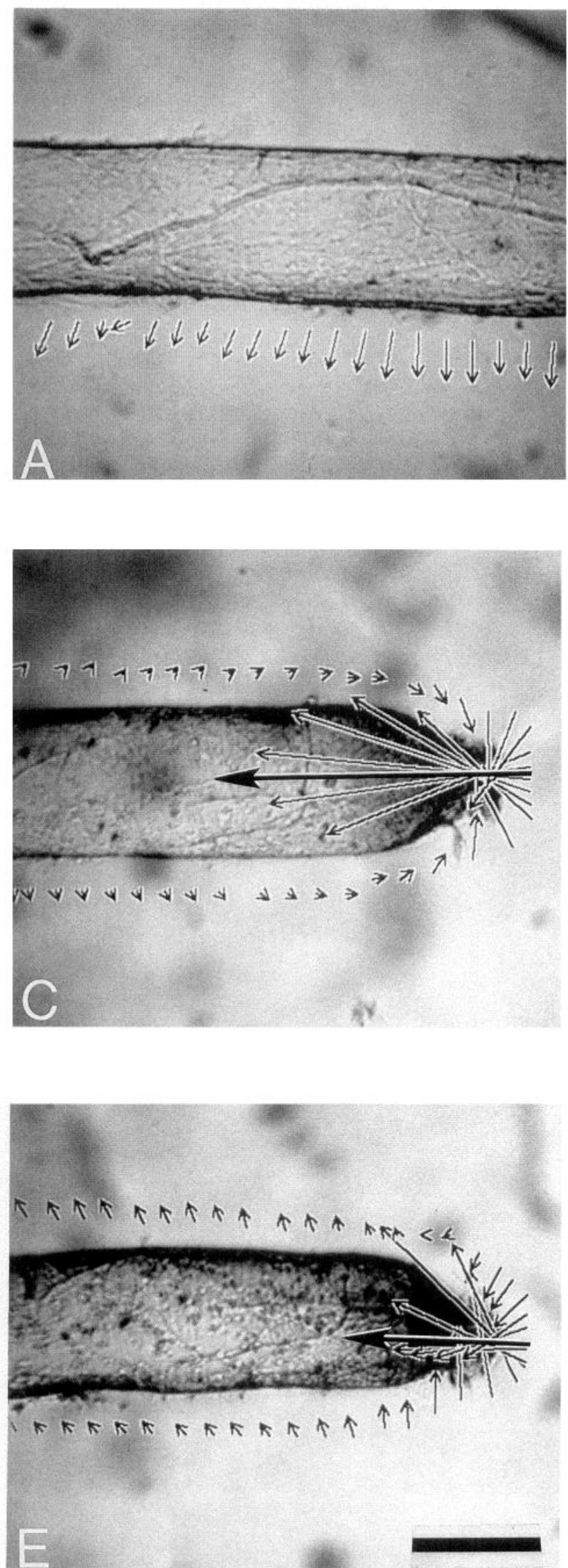

\section{Earthworm MGA}
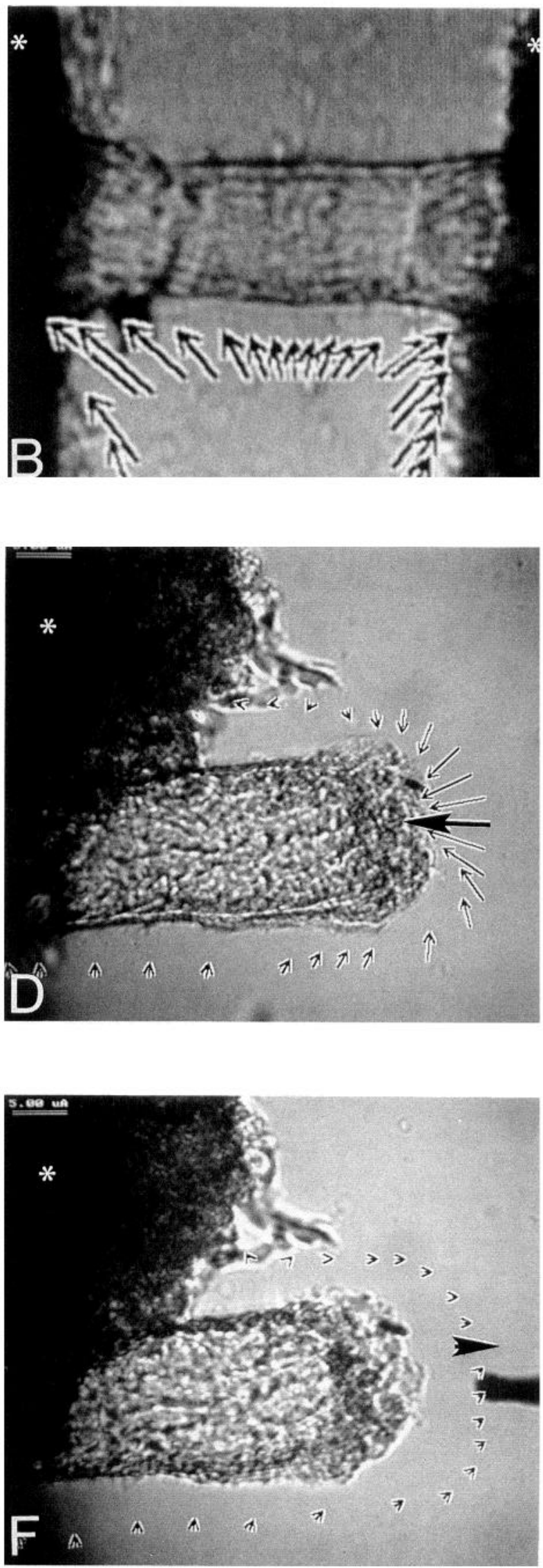
small inward control current density vectors between two pieces of ventral nerve cord (Fig. $2 B$ ). The cut ends of ventral nerve cords (asterisks) of earthworm had large inward control current density vectors. The greater amount of damaged neuronal tissue attached to dissected MGAs compared to dissected GAs probably accounted for the greater magnitude and inward direction of control current densities in MGAs.

After transection, the spatial distribution of $I_{\text {inj }}$ along an axon and at its cut end was characterized for several GAs $(n=4)$ and MGAs $(n=4)$ by placing the vibrating probe the fixed distance from the surface of the axon at various points along its length and its cut end (Fig. 2C-F). Transection of GAs and MGAs in standard salines created a large net inward $I_{\mathrm{inj}}$ at the cut ends (Fig. $2 C-F$ ). The largest $I_{\text {inj }}$ vector was always perpendicular to the cut axonal end (Fig. $2 C-E$, thick arrows). For squid GAs examined up to $2.5 \mathrm{hr}$ after transection in standard ASW, the magnitude of $I_{\text {inj }}$ vectors was always greater at the cut axonal end compared to other sites along the axon (Fig. 2C,E). Likewise, for earthworm MGAs examined up to $30 \mathrm{~min}$ after transection in standard EWS, $I_{\text {inj }}$ magnitude was always greater at the cut axonal end compared to other sites along the axon (Fig. $2 D$ ). In contrast, for MGAs examined at $60 \mathrm{~min}$ after axonal transection (Fig. 2F) in standard EWS, the magnitude of $I_{\text {inj }}$ vectors at the cut axonal end was the same as $I_{\text {inj }}$ vectors measured at other sites along the axon. The $I_{\text {inj }}$ vectors at $60 \mathrm{~min}$ were not always directed inward at the cut end for earthworm MGAs (Fig. $2 F$ ) as were $I_{\text {inj }}$ vectors in squid GAs measured up to $2.5 \mathrm{hr}$ after transection (Fig. $2 C, E$ ) or in earthworm MGAs measured $\leq 3 \mathrm{~min}$ after transection (Fig. $2 D$ ).

In addition to evaluating the time course and extent of sealing by comparing $I_{\mathrm{inj}}$ at the cut end with $I_{\mathrm{inj}}$ along the membrane of the cut axon (Fig. $2 C-F$ ), we compared the average control current density (Fig. $2 A, B$ ) of intact axons with the maximum net $I_{\text {inj }}$ parallel to the longitudinal axis of the axon $\left(I_{\max }\right)$ (Fig. $2 C-F$, thick arrows) at the fixed distance from the cut end. We used $I_{\max }$ as a single measure to characterize $I_{\text {inj }}$ because we were able to perform this single measurement much more quickly compared to the time required to make several measurements along the axon.

After measuring control current densities of several squid GAs $(n=3)$ in standard ASW (Table 1), GAs were transected in standard ASW $(n=4)$ or divalent cation-free ASW $(n=4) . I_{\max }$ measured at $\leq 8 \mathrm{~min}$ after transection in standard ASW was about 100 times greater than control current densities measured in intact axons. Although the $I_{\max }$ had declined to less than $20 \%$ of this initial value by $2.5 \mathrm{hr}$ after transection, the $I_{\max }$ at $2.5 \mathrm{hr}$ was still over 14 times greater than control current density measured in intact GAs (Table 1; compare rows 1-3). This $I_{\max }$ at $2.5 \mathrm{hr}$ in standard ASW was significantly different from control current density ( $P<0.05$, Student's $t$ test). In contrast, when GAs were bathed in divalent cation-free ASW, $I_{\max }$ at $\leq 8 \mathrm{~min}$ after transection was 160 times greater than control current density in intact axons bathed in standard ASW. The greater initial $I_{\max }$ values in divalent cation-free ASW versus standard ASW (Table 1; compare rows 2 and 4) are probably due to differences (i.e., diameter, $v_{m}$ ) in the axons used in these two studies, rather than a rapid effect of the salines. Indeed, the initial $I_{\max }$ was very similar when segments of the same GA were bathed in standard $\left(207.8 \pm 3.5 \mu \mathrm{A} / \mathrm{cm}^{2}\right)$ or divalent cationfree saline $\left(226.6 \pm 10.6 \mu \mathrm{A} / \mathrm{cm}^{2}\right)(n=2)$. At $2.5 \mathrm{hr}$ after transection, $I_{\max }$ for squid GAs maintained in divalent cation-free ASW had declined to $37 \%$ of the initial value and was 60 times greater than control current density in intact axons.
After measuring control current densities of several earthworm MGAs $(n=7)$ in standard EWS (Table 1), MGAs were transected in divalent cation-free EWS $(n=10)$ since other data indicated that MGAs seal very rapidly in standard EWS (see Figs. $1 B ; 4 F, H ; 7$; see also Discussion) but not in divalent cationfree EWS (Fig. $4 B$; see Discussion). The initial $I_{\max }$ measured at $\leq 8$ min after transection in divalent cation-free EWS was over six times greater than control current densities measured in standard EWS. In some cases, divalent cation-free EWS was replaced with standard EWS $(n=6)$ over a $3 \mathrm{~min}$ period. In these MGAs, $I_{\max }$ at 45 min in standard EWS was $13 \%$ of the $I_{\max }$ measured prior to the solution change from divalent cationfree EWS to standard EWS, and less than values of control current density (Table 1). This $I_{\max }$ at $45 \mathrm{~min}$ in standard saline was not significantly different $(P<0.05$, Student's $t$ test) from the control current density measured in intact MGAs. In contrast, transected MGAs maintained in divalent cation-free EWS $(n=4)$ had an $I_{\max }$ at $45 \mathrm{~min}$, which was more than $60 \%$ of the initial $I_{\max }$ and $460 \%$ of the control current density of intact MGAs in standard EWS (Table 1). These data strongly suggest that large reductions in extracellular calcium prevent the sealing of transected earthworm MGAs.

To facilitate comparison of $I_{\max }$ changes in GAs versus MGAs shown in Figure 3, we normalized the $I_{\max }$ for each axon by setting the initial $I_{\max }$ to $100 \%$ and the control current density to $0 \%$. According to this normalized measure, $I_{\max }$ would always equal $100 \%$ at $8 \mathrm{~min}$ after transection and would decline to $0 \%$ only if the axon formed a seal whose current leak was equal to that across an equivalent membrane area from an intact axon.

As plotted in Figure 3, the decline of initial $I_{\max }$ was quite different for squid GAs versus earthworm MGAs. For example, $I_{\max }$ declined to levels of control current density within $45 \mathrm{~min}$ in MGAs, but did not reach levels of control current density by $2.5 \mathrm{hr}$ in GAs. These data suggest that MGAs, but not GAs, form a functional seal within several hours after transection when bathed in standard salines. In contrast, the decline of initial $I_{\max }$ was much less when either axon was transected and maintained in divalent cation-free saline, suggesting that neither axon seals in a divalent cation-free medium. In fact, the decline of $I_{\max }$ in squid GAs and earthworm MGAs transected in divalent cation-free saline almost certainly represents the deterioration of axons (e.g., the decline of ionic driving forces at the cut ends), rather than the formation of a seal.

\section{Structural changes of transected GAs and MGAs assessed in vitro}

Within 5 min of transection in standard ASW, squid GAs ( $n=$ 6), began to constrict at their cut ends, contract along their long axes, discharge axoplasm from the cut ends, and form intraaxonal vesicles. At $5 \mathrm{~min}$ after transection, the cut ends of GAs had constricted to $57 \%(280 \pm 20 \mu \mathrm{m}, \pm$ SEM) of their original diameters $(490 \pm 50 \mu \mathrm{m})$, and the point of maximal constriction was $100-300 \mu \mathrm{m}$ from the cut end. Vesicles, typically $1-10 \mu \mathrm{m}$ in diameter, were present along the axolemma for several hundred micrometers from the cut end. The density of these vesicles along the axolemma was greatest near the cut end, becoming progressively less dense away from the cut.

During the next $2.5 \mathrm{hr}$, GAs continued to constrict at their cut ends and contracted along their long axes by $200-1000 \mu \mathrm{m}$. Similar contraction following axonal transection has been routinely observed in a variety of vertebrate axons (Ramon y Cajal, 1928; Bird, 1978; Sole, 1980; Lucas, 1987; George et al., 1988). Additionally, the intraaxonal vesicles grew in number and size- 


\begin{tabular}{|c|c|c|}
\hline $\begin{array}{l}\text { Experimental } \\
\text { conditions }\end{array}$ & $\begin{array}{l}\text { Squid GAs } \\
\left(\mu \mathrm{A} / \mathrm{cm}^{2}\right)\end{array}$ & $\begin{array}{l}\text { Earthworm MGAs } \\
\left(\mu \mathrm{A} / \mathrm{cm}^{2}\right)\end{array}$ \\
\hline \multicolumn{3}{|c|}{ Physiological salines } \\
\hline Control current & $2.6 \pm 0.4 \quad(n=3)$ & $10.1 \pm 1.8 \quad(n=7)$ \\
\hline Initial $I_{\mathrm{inj}}$ & $179.9 \pm 48.1(n=4)$ & $72.1 \pm 13.9(n=6)^{b}$ \\
\hline Final $^{a} I_{\text {inj }}$ & $27.6 \pm 6.4 \quad(n=4)$ & $9.3 \pm 3.7 \quad(n=4)$ \\
\hline \multicolumn{3}{|c|}{ Divalent cation-free salines } \\
\hline Initial $I_{\text {inj }}$ & $323.9 \pm 50.8(n=4)$ & $66.6 \pm 22.3(n=4)$ \\
\hline Final $^{a} I_{\text {inj }}$ & $120.8 \pm 21.8(n=4)$ & $45.8 \pm 20.6(n=4)$ \\
\hline
\end{tabular}

${ }^{a}$ Final $I_{\mathrm{inj}}$ measured at $2.5 \mathrm{hr}$ for GAs and 45 min for MGAs.

${ }^{b}$ Measured in divalent cation-free EWS due to rapid sealing of MGAs in standard EWS.

often migrating to the cut end and sometimes fusing with each other. At $2.5 \mathrm{hr}$ after transection in standard ASW, the cut ends of GAs had constricted to $5-15 \%(40 \pm 10 \mu \mathrm{m})$ of their original diameters, with the point of maximal constriction $100-300 \mu \mathrm{m}$ from the cut end (Fig. $4 C$ ). That is, at $2.5 \mathrm{hr}$ after transection in standard ASW, the diameter of the opening at the cut end was about $40 \mu \mathrm{m}$ at its narrowest point. At $2.5 \mathrm{hr}$, many vesicles (typically $20-50 \mu \mathrm{m}$ in diameter) were present along the axolemma and in the axoplasm of the GA for several hundred micromcters from the cut end (Fig. 4E). Vesicle density along the axolemma and in the axoplasm was greatest near the cut end. Large ( $\geq 50 \mu \mathrm{m}$ ) vesicles sometimes protruded from, and appeared to occlude, the cut end (Fig. $4 G$ ). Sequential "optical sections" using VE-DIC indicated that there were typically tens of micrometers between the vesicles near the cut end-even at the point of maximal constriction. At no time following transection in standard saline did we observe any structures or axoplasmic discontinuities that spanned the diameter of the GA at the cut end or within several millimeters from the cut axonal end.

Within 5 min of transection in standard EWS, earthworm MGAs $(n=7)$ began to constrict at their cut ends, contract along their long axes, discharge small amounts of axoplasm from the cut ends, and form intraaxonal vesicles. In other experiments $(n>20)$, the addition of measured amounts of calcium or standard EWS to divalent cation-frec EWS cnabled us to control the initiation and speed of constriction (Gallant, 1988) and vesicle formation (Fishman et al., 1990) in transected MGAs. Unlike squid GAs, most constriction at the cut ends of earthworm MGAs took place in the first minute after exposure of the cut end to standard EWS. A rapid movement of axoplasm, vesicles, and other debris toward the transected end occurred within the first $30 \mathrm{sec}$ of exposing the cut end to standard EWS. Al $5 \mathrm{~min}$ after transection in EWS, the cut ends of MGAs had constricted to $52 \%(44 \pm 6 \mu \mathrm{m})$ of their original diameters $(85 \pm 4 \mu \mathrm{m})$; the point of maximal constriction was at the cut end. Intraaxonal vesicles primarily $5-30 \mu \mathrm{m}$ in diameter were observed along the axolemma, in the axoplasm, and throughout the axonal sheath (Fig. $4 F$ ). The density of these vesicles along the axolemma and in the axoplasm was greatest near the cut end, becoming progressively less dense away from the cut.

During the next hour, MGAs continued to constrict at their cut ends and contracted along their long axes by $20-150 \mu \mathrm{m}$. The intraaxonal vesicles grew in number and size, often migrating to the cut end. These vesicles were sometimes directly observed with VE-DIC to fuse with each other. At $1 \mathrm{hr}$ after
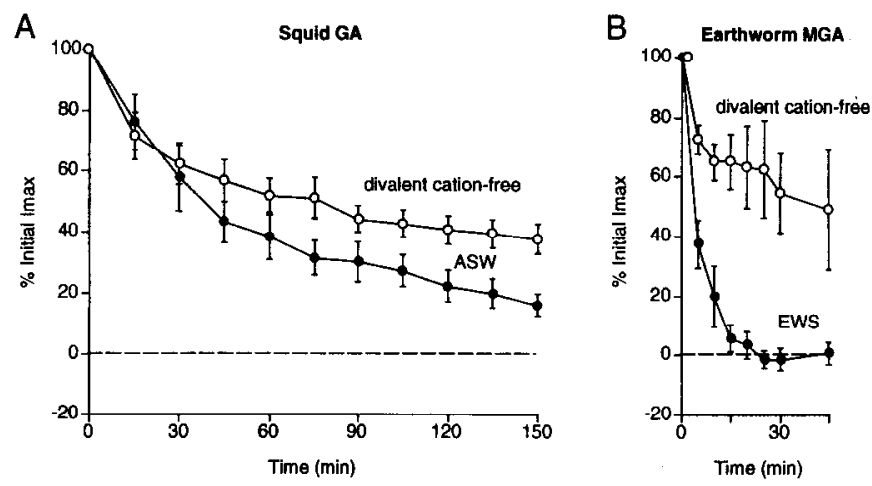

Figure 3. Variation of $I_{\max }$ with time after transection of squid GAs and earthworm MGAs in standard salines (solid circles) and divalent cation-free salines (open circles). Initial $I_{\max }(0 \mathrm{~min})$ set to $100 \%$ and control current density (dashed lines) set to $0 \%$. Maximum net current density measured parallel to the longitudinal axis of transected axons $\left(I_{\max }\right)$ is compared to the average current density measured the same distance from intact (control) axons. A, Squid (Loligo pealei) GAs transected in standard saline (solid circles; $n=4$ ) or divalent cation-free saline (open circles; $n=4$ ) and monitored for $150 \mathrm{~min}$ after the initial measure of $I_{\max }$. $B$, Earthworm MGAs transected in standard saline (solid circles; $n=6$ ) or divalent cation-free saline (open circles; $n=4$ ) and monitored for $45 \mathrm{~min}$ after the initial measure of $I_{\max }$. Note that $I_{\max }$ did not decline to control values in squid GAs within $2.5 \mathrm{hr}$, but did decline to control values in earthworm MGAs within 30 min. Further, $I_{\max }$ declined when GAs and MGAs were bathed in divalent cationfree salines, presumably by slow axonal depolarization rather than by axonal sealing.

transection in standard EWS, the cut ends had constricted to $34 \%(37 \pm 6 \mu \mathrm{m})$ of their original diameters, with the point of maximal constriction at the cut end (Fig. 4D). That is, at $1 \mathrm{hr}$ after transection, MGA axonal diameter was about $40 \mu \mathrm{m}$ at its narrowest point. Further, at $1 \mathrm{hr}$, vesicles, distributed in size from 5 to $30 \mu \mathrm{m}$, were present along the axolemma and in the axoplasm of the MGA. Vesicle density near the cut end was much greater than away from the cut end and large $(\geq 50 \mu \mathrm{m})$ vesicles often protruded from, and appeared to occlude, the cut end (Fig. $4 H$ ). [These large vesicles were only located at or near $(\leq 50 \mu \mathrm{m})$ the cut end and did not appear to arise from septal membranes.] Unlike vesicles in the squid GA, sequential "optical sections" using VE-DIC indicated that vesicles were densely packed only at the point of maximal constriction (i.e., vesicles filled the constricted cut end regardless of the plane of focus). We never observed any continuous structures or axoplasmic discontinuities that spanned the diameter of the MGA at the cut end or within several millimeters from the cut end.

Following transection in divalent cation-free salines (1-3 hr), squid GAs $(n=7)$ and earthworm MGAs $(n=5)$ neither constricted at their cut ends, nor discharged axoplasm from the cut axonal end, nor contracted longitudinally, nor formed large ( $\geq 5$ $\mu \mathrm{m}$ ) intra-axonal vesicles (Fig. $4 A, B$ ). That is, there were no gross structural differences between intact axons and axons transected and maintained for up to $2.5 \mathrm{hr}$ in divalent cation-free saline. These data are consistent with our functional measures of $v_{m}, R_{\mathrm{in}}$, and $I_{\mathrm{inj}}$, which suggest that transected squid GAs and earthworm MGAs do not seal when extracellular calcium is greatly reduced.

\section{Structural changes of transected GAs and MGAs assessed in fixed material}

In agreement with previous reports (Villegas and Villegas, 1984; Gilbert et al., 1990), we found that intact squid GAs typically ranged from 300 to $600 \mu \mathrm{m}$ in diameter and were surrounded 


\section{Squid GA}
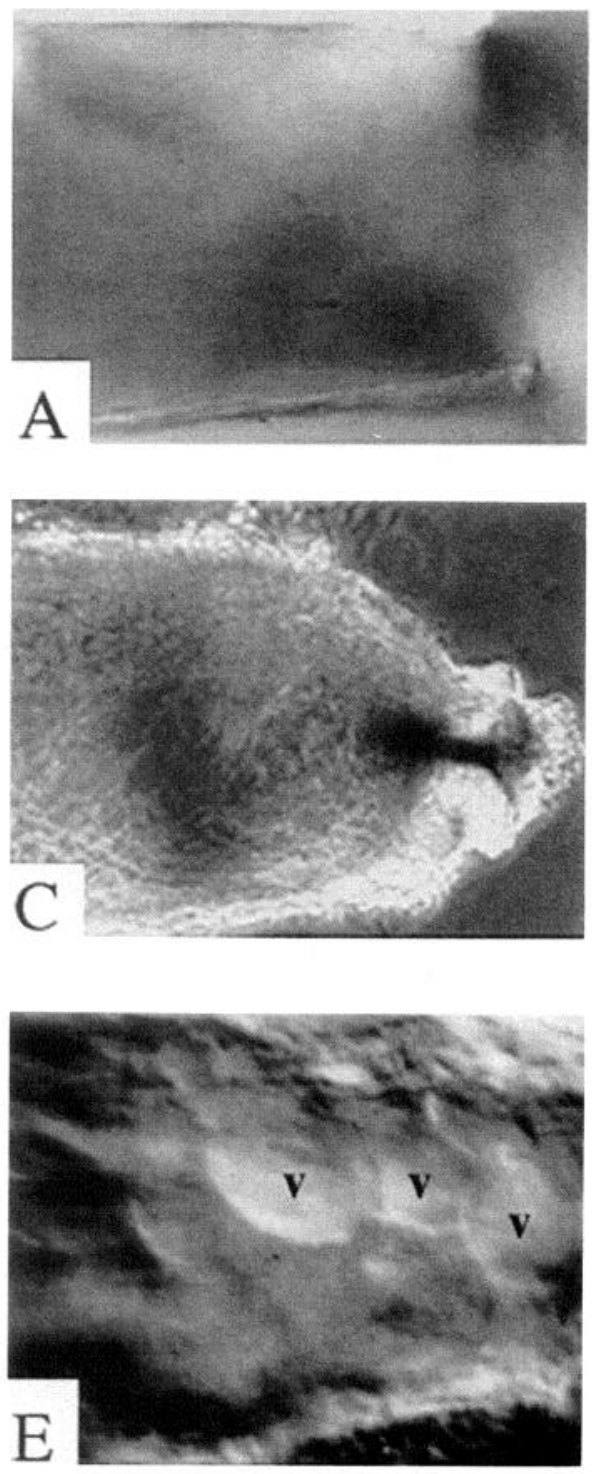

Figure 4. Transected GAs (left column) and MGAs (right column) viewed with phase-contrast and VE-DIC microscopy. Cut ends of transected GAs (Loligo pealei) and MGAs are to the right in each micrograph in this and other figures. $A$ and $B$, Axons in divalent cation-free salines $15 \mathrm{~min}$ posttransection. Note that the cut axonal ends of the GA $(A)$ and MGA $(B)$ are not constricted and contain no intraaxonal vesicles. $C$ and $D$, Squid $\mathrm{GA} 3 \mathrm{hr}$ (C) and earthworm MGA $1 \mathrm{hr}(D)$ after transection in standard salines. Note that the cut end of the squid GA is considerably constricted $(C)$, whereas the cut end of the earthworm MGAs is slightly constricted $(D)$ ( $v$, injury-induced vesicles). $E$ and $F$, Vesicles accumulated near cut ends of a GA $1 \mathrm{hr}$ $(E)$, and an MGA $10 \mathrm{~min}(F)$, after transection in standard salines. $G$ and $H$, Vesicles protruding from cut ends of a squid GA $1.5 \mathrm{hr}(G)$, and an earthworm MGA 6 min $(H)$, after transection in standard salines. Scale bar: $A, 300 \mu \mathrm{m}$; $B, 60 \mu \mathrm{m} ; C, 275 \mu \mathrm{m} ; D, 30 \mu \mathrm{m} ; E, 75$ $\mu \mathrm{m} ; F, 80 \mu \mathrm{m} ; G, 140 \mu \mathrm{m} ; H, 50 \mu \mathrm{m}$.

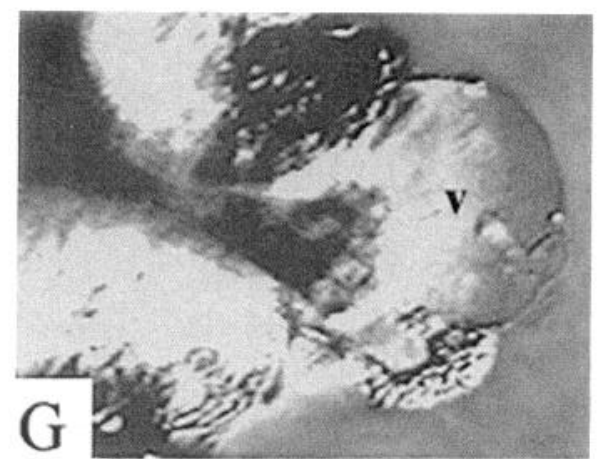

\section{Earthworm MGA}
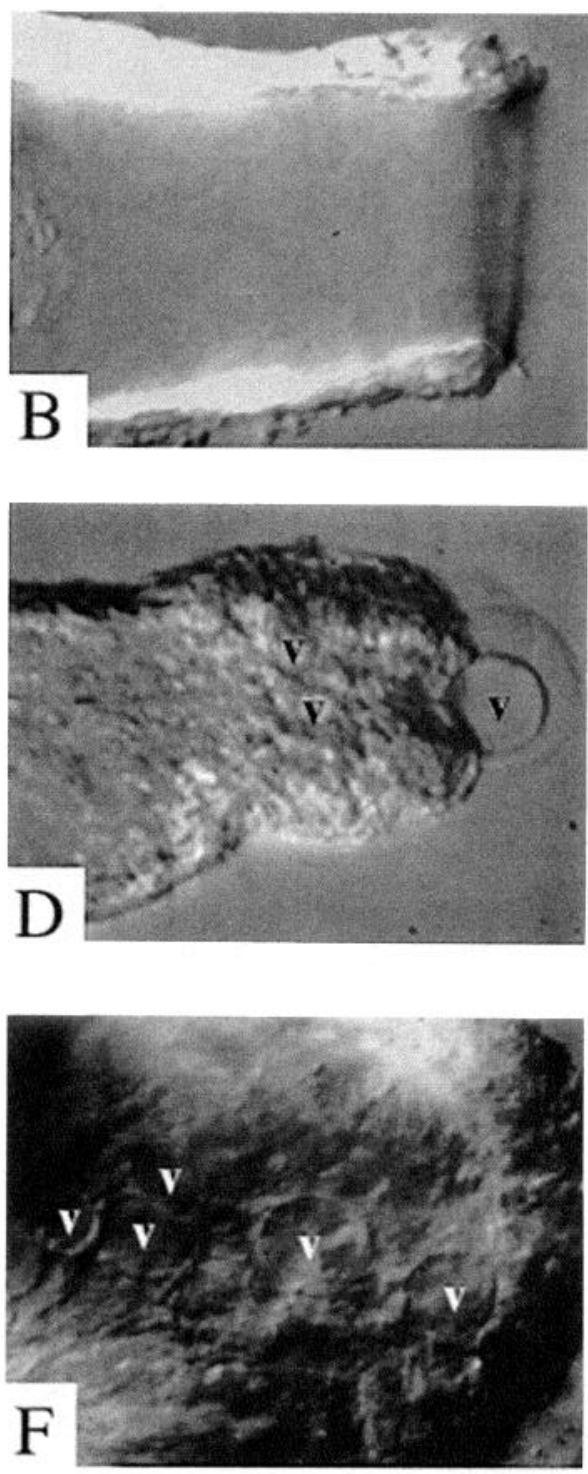

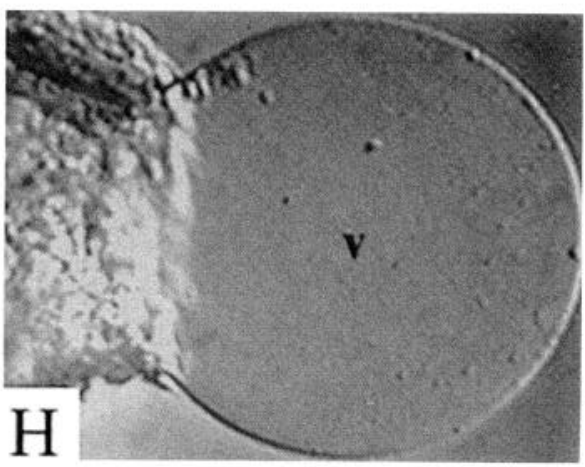

by an adaxonal glial layer 1-2 $\mu \mathrm{m}$ thick bounded by a basement membrane (Fig. $5 \mathrm{~A}$ ). When viewed at higher magnifications the axoplasm of typical squid GAs contained mitochondria, neurofilaments, microtubules, smooth endoplasmic reticulum, and vesicles (data not shown). The adaxonal glia contained mitochondria, microtubules, rough and smooth endoplasmic retic- ulum, and vesicles. [In this article the term "vesicle" refers to any structure surrounded by a single membrane in the axoplasm or gliaplasm.] This glial layer was bounded by a continuous basement membrane (Fig. $5 C$ ). Squid GAs fixed 5 min after transection $(n=3)$ were constricted at their cut ends to $39 \%$ $(160 \pm 10 \mu \mathrm{m})$ of their control diameters $(410 \pm 10 \mu \mathrm{m})$ mea- 

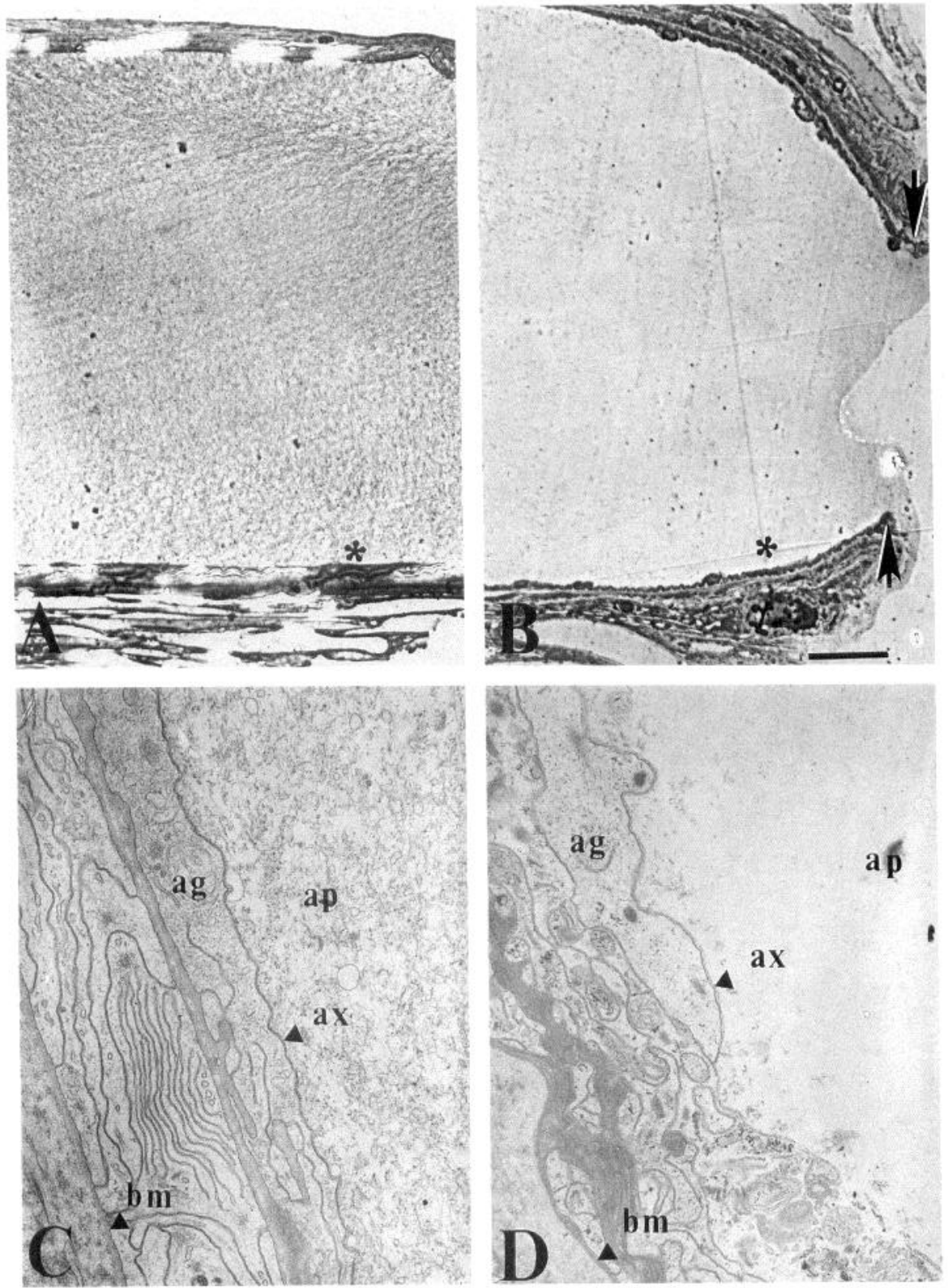

Figure 5. Light $(A, B)$ and electron $(C$, $D$ ) micrographs of squid GAs fixed intact $(A, C)$ and at 5 min posttransection $(B, D)$. GAs (Sepioteuthis lessoniana) are longitudinally sectioned in all micrographs. $C$ and $D$ are from areas $\left(^{*}\right)$ of $A$ and $B$, respectively. Note that the GA fixed at 5 min posttransection $(B)$ is constricted at its cut end, and glia near its cut end are swollen (compare $A$ and $C$ with $B$ and $D$ ). Abbreviations for Figures 5-8: $a p$, axoplasm; $a g$, adaxonal glia; $a x$, axolemma; $b m$, basement membrane; facing arrows, cut site. Scale bar: $A$ and $B, 50 \mu \mathrm{m} ; C$ and $D, 2$ $\mu \mathrm{m}$. sured as the diameter $0.5 \mathrm{~mm}$ from the cut site. The point of maximal constriction was typically within $50-100 \mu \mathrm{m}$ from the cut end. The axoplasm was similar to that in intact axons, except for mitochondrial swelling. Vesicles were seen near the cut axonal end. The adaxonal glial layer had an increased thickness within $100 \mu \mathrm{m}$ from the cut end (compare Fig. 5, $C$ and $D$ ); mitochondria and other membrane bound organelles were noticeably swollen. Squid GAs fixed $2.5 \mathrm{hr}$ after transection $(n=$ 3) constricted at their cut ends to $20 \%(70 \pm 15 \mu \mathrm{m})$ of their control diameters $(350 \pm 40 \mu \mathrm{m})$. The point of maximal constriction was typically $100-300 \mu \mathrm{m}$ from the cut end (Fig. 6A). Many vesicles were present in the axoplasm and glial sheath within 1-2 $\mathrm{mm}$ of the cut end (Fig. $6 A-C$ ). Although some vesicles were as large as $30 \mu \mathrm{m}$, most vesicles ranged from 0.01 to $2 \mu \mathrm{m}$ in diameter. Vesicles near the cut end were loosely spaced in translucent axoplasmic debris and no continuous membrane barriers were observed (Fig. 6C).

In agreement with previous reports (Coggeshall, 1965; Günther, 1976; Birse and Bittner, 1981), we found that intact MGAs typically ranged from 80 to $100 \mu \mathrm{m}$ in diameter and were surrounded by an invertebrate-myelin sheath 3-9 $\mu \mathrm{m}$ thick (Fig. $7 A$ ). When viewed at higher magnifications the axoplasm of control MGAs contained mitochondria, neurofilaments, microtubules, and vesicles (data not shown). The glial sheath consisted primarily of compact layers of invertebrate-myelin (Fig. 7C). Earthworm MGAs fixed 5 min after transection $(n=3)$ were constricted at their cut ends to $45 \%(50 \pm 15 \mu \mathrm{m})$ of their control diameters $(112 \pm 21 \mu \mathrm{m})$ (Fig. $7 B$ ). The cut end was filled with vesicles $1-20 \mu \mathrm{m}$ in diameter and some axoplasmic debris. Within $500 \mu \mathrm{m}$ of the cut end, vesicles were obviously present in the cortical regions of the axoplasm and the adaxonal regions of the glial sheath (Fig. $7 B, D$ ). Furthermore, axoplasmic neurofilaments and microtubules were disrupted and axoplasmic mitochondria were swollen. Earthworm MGAs fixed $60 \mathrm{~min}$ after transection $(n=5)$ were constricted at their cut ends to $68 \%$ $(61 \pm 12 \mu \mathrm{m})$ of their control diameters $(90 \pm 7 \mu \mathrm{m})$ (Fig. 8A). Although no continuous membrane barrier was observed, the cut end was filled with vesicles $1-20 \mu \mathrm{m}$ in diameter (Fig. $8 A, C$ ). 
Figure 6. Light $(A)$ and electron $(B$, $C$ ) micrographs of a squid GA fixed 2.5 $\mathrm{hr}$ posttransection. The squid (Sepioteuthis lessoniana) GA is longitudinally sectioned in all micrographs. $B$ and $C$ are from areas $\left({ }^{*}\right)$ of $A$. Note that the cut end is constricted $(A)$, glia are vesiculated $(B)$, and small intra-axonal vesicles can be seen along the axolemma $(A, B)$ and at the cut end $(A, C) . v$, vesicle; $S$, sheath; other details are as for Figure 5. Scale bar: $A, 50 \mu \mathrm{m} ; B$ and C, $5 \mu \mathrm{m}$.
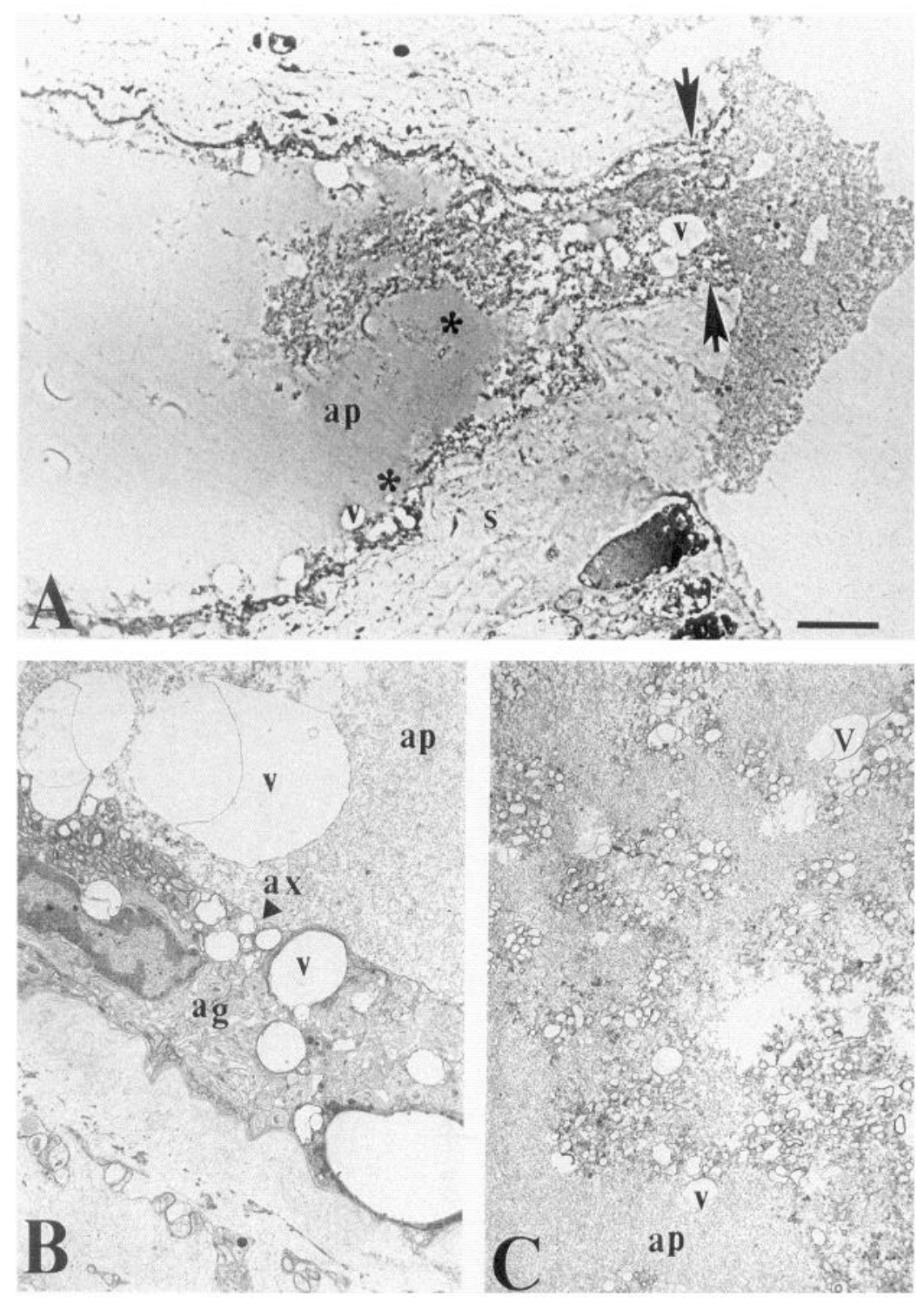

Compared to vesicles seen at the cut end of MGAs fixed 5 min posttransection (Fig. 7D), these vesicles were typically smaller and were tightly packed in electron opaque axoplasmic debris (Fig. $8 C$ ). Vesicles were also present in the cortical layers of axoplasm and within the invertebrate-myelin sheath (Fig. 8B). The invertebrate-myelin layers of the sheath were very loose and disorganized near the cut end (Fig. $8 B$ ).

\section{Discussion}

To prevent the toxic changes in the internal concentrations of ions and macromolecules, an axonal seal should be a highresistance barrier that reduces the net ionic current at the cut end to values observed across an equivalent area of intact axolemma. Functional measures (e.g., $v_{m}, R_{\mathrm{in}}, I_{\mathrm{inj}}$ ) used to assess an axonal seal should accurately reflect the resistance or ionic currents at the cut end. Morphological mechanisms (e.g., axoplasmic constriction followed by axolemmal fusion) proposed to account for sealing should be consistent with temporal changes in functional measures of sealing. No previous study has shown a continuous membrane barrier at the cut end, much less how the formation of such a barrier correlates with changes in appropriate functional measures of sealing.

\section{Functional measures of the extent of seal formation}

Restoration of $v_{m}$ and/or $R_{\text {in }}$ have been cited more frequently than any other functional measures to assess the extent of seal formation (Weidmann, 1952; Deleze, 1970; De Mello, 1973; Meiri et al., 1981; Yawo and Kuno, 1983, 1985; Lucas et al., 1985; Spira et al., 1993). These previous studies have assumed that a recovery of $v_{m}$ and/or $R_{\text {in }}$ to control or predicted values indicates complete sealing of the cut end. However, measures of $v_{m}$ and $R_{\text {in }}$ are influenced by many factors other than the extent of seal formation. For example, $v_{m}$ depends on the distribution of potential along the fiber and thus on the electrotonic distance between the site of measurement and the cut axonal end (Jack et al., 1983). Similarly, $R_{\text {in }}$ depends on the current distribution along the fiber and thus on the electrotonic distance between the site of measurement and the cut axonal end (Weidmann, 1952; Jack et al., 1983). Electrotonic distance and electrode placement usually cannot be accurately determined prior to transection - much less after transection. That is, electrotonic 

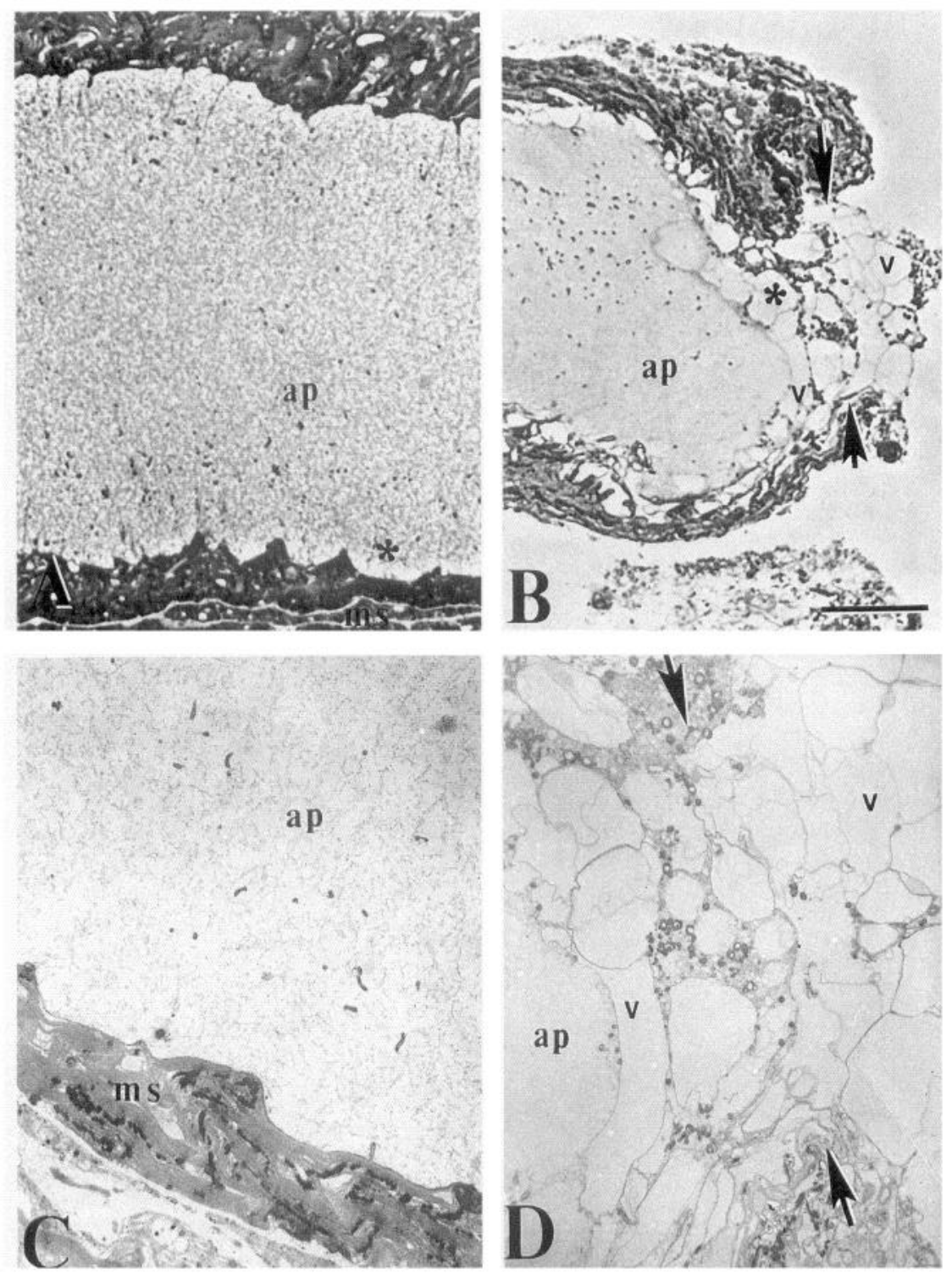

Figure 7. Light $(A, B)$ and electron $(C$, $D)$ micrographs of earthworm MGAs fixed intact $(A, C)$ and at $5 \mathrm{~min}$ posttransection $(B, D)$. MGAs are longitudinally sectioned in all micrographs. $C$ and $D$ are from areas (*) of $A$ and $B$, respectively. Note that the cut end of the MGA fixed at $5 \mathrm{~min}$ posttransection $(B)$ is slightly constricted and contains an accumulation of large vesicles. Note also that glia are swollen and vesiculated in the MGA fixed $5 \mathrm{~min}$ posttransection (compare $A$ and $C$ with $B$ and $D$ ). $v$, vesicle; $m s$, (invertebrate) myelin sheath; other details are as for Figure 5. Scale bar: $A, 20 \mu \mathrm{m} ; B, 30 \mu \mathrm{m}$; $C$ and $D, 6.5 \mu \mathrm{m}$.

distance can change by as much as $50 \%$ after transection (Yawo and Kuno, 1985), possibly due to axonal constriction (Gallant, 1988 ) and/or a decrease in $v_{m}$, which can activate voltage-sensitive channels that were not all blocked in previous studies.

We measured $v_{\mathrm{m}}$ and $R_{\text {in }}$ to compare our data from squid GAs and earthworm MGAs with data from previous reports concluding that their data were consistent with axonal sealing. We find that $v_{\mathrm{m}}$ recovers in transected squid GAs to about $70 \%$ of control values within $1.5 \mathrm{hr}$ after transection and slowly declines thereafter. This inability of $v_{m}$ to recover to control levels within $1 \mathrm{hr}$ is similar to findings in transected cockroach giant axons (Meiri et al., 1981; Leech and Treherne, 1984) and giant axons in the lamprey spinal cord (Strautman et al., 1990). We find that $v_{m}$ recovers in transected earthworm MGAs to $90-100 \%$ of control values within $1 \mathrm{hr}$ after transection. This ability of $v_{m}$ to recover in earthworm MGAs is similar to that reported for transected cockroach giant axons (Yawo and Kuno, 1983, 1985), for transected dendrites of cultured mouse neurons (Lucas et al., 1985), and for severed Aplysia axons in tissue culture (Spira et al., 1993). We find that $R_{\text {in }}$ in transected GAs and MGAs recovers within $1-2.5 \mathrm{hr}$ to exceed control $R_{\text {in }}$ values by $135 \%$ in GAs and 22\% in MGAs. The time course of $R_{\text {in }}$ recovery in GAs and MGAs is similar to that measured in cockroach giant axon by Yawo and Kuno $(1983,1985)$, but is slower than the recovery period reported for Aplysia axons severed in tissue culture (Spira et al., 1993). In brief, the changes we observed in $v_{m}$ and $R_{\text {in }}$ for both squid GAs and earthworm MGAs are similar to changes previously reported for other preparations that have been reported to seal as measured by the recovery of $\mathrm{v}_{\mathrm{m}}$ and $R_{\text {in }}$. However, as discussed above, measures of $v_{m}$ and $R_{\text {in }}$ cannot accurately assess the extent of sealing.

Since $I_{\text {inj }}$ is an extracellular measure of seal formation that is free of many of the artifacts discussed above for intracellular measures $\left[v_{m}, R_{\text {in }}\right]$ of seal formation, we assessed $I_{\text {inj }}$ in two ways: (1) the magnitude and direction of $I_{\text {inj }}$ vectors at cut axonal ends were compared to vectors measured elsewhere along the axolemma of the same axons, and (2) the magnitude of $I_{\max }$ vectors at cut axonal ends were compared to the magnitude of control current densities along intact axons. Both measures assume an axon is completely sealed when the cut axonal end has the same current density as the axolemma. According to both of these measures, $I_{\text {inj }}$ at the cut end of squid GAs in standard ASW does 

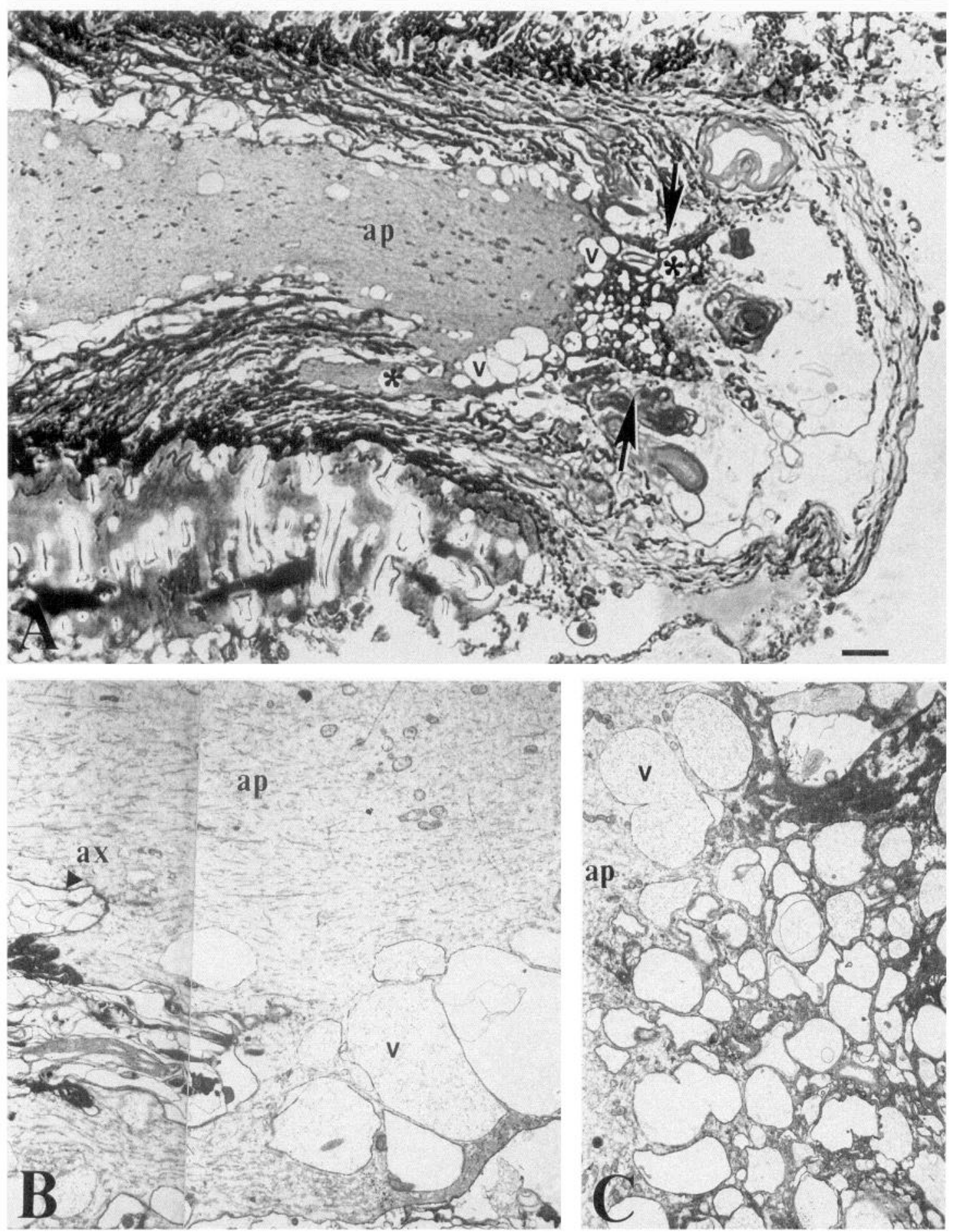

Figure 8. Light $(A)$ and electron $(B, C)$ micrographs of a typical earthworm MGA fixed $1 \mathrm{hr}$ posttransection. The MGA is longitudinally sectioned in all micrographs. $B$ and $C$ are from areas $\left(^{*}\right)$ of $A$. Note that the cut end is slightly constricted $(A)$, glia are vesiculated $(B)$, and large intra-axonal vesicles can be seen along the axolemma $(A, B)$ and accumulated at the cut end $(A, C), v$, vesicle; other details are as for Figure 5 . Scale bar: $A$, 10 $\mu \mathrm{m} ; B$ and $C, 3 \mu \mathrm{m}$.

not decline to the level of current density along the axolemma of either transected or intact axons within $2.5 \mathrm{hr}$ of transection. In fact, $2.5 \mathrm{hr}$ after GA transection, $I_{\mathrm{inj}}$ is $10-20$ times the control current density along the axolemma of intact axons. These data indicate that the cut ends of squid GAs have a much lower resistance to ion flow within $2.5 \mathrm{hr}$ after transection than the intact axolemma. In contrast, both of these $I_{\text {inj }}$ measures show that $I_{\mathrm{inj}}$ at the cut end of earthworm MGAs in standard EWS declines within $1 \mathrm{hr}$ to equal the current density along the axolemma of transected or intact axons. These data suggest that within $1 \mathrm{hr}$ after transection, the cut end and the axolemma of the MGA have the same resistance to ionic current flow.

Although $I_{\text {inj }}$ is arguably the best measure of the extent of seal formation, measures of $I_{\text {inj }}$ alone cannot unambiguously assess 
the extent of axonal sealing. $I_{\text {inj }}$ will decline as an axon progressively depolarizes, even if the axon does not seal. Hence, a large decline of $I_{\text {inj }}$ cannot distinguish between an axon that has completely sealed and an axon that has completely depolarized. Further, currents produced by tissue surrounding the axon could obscure the evaluation of sealing using $I_{\text {inj }}$ measures alone. Measures of $v_{\mathrm{m}}$ and $R_{\mathrm{in}}$ can help distinguish the time course and extent of sealing in such situations. Hence, consideration of $v_{\mathrm{m}}$, $R_{\text {in }}$, and $I_{\text {inj }}$ data allows a more definitive assessment of the time course and extent of sealing.

Our data from all three functional measures of seal formation ( $v_{m}, R_{\text {in }}$, and $I_{\text {inj }}$ ) for squid GAs are consistent with the conclusion that GAs do not seal within $2.5 \mathrm{hr}$ after transection in standard salines, that is, $v_{m}$ and $I_{\mathrm{inj}}$ do not return to values recorded in intact axons. Although $R_{\text {in }}$ increases to values greater than recorded for intact axons, this increase could be due to axonal changes that are not associated with the formation of a continuous seal such as changes in axon diameter resulting from constriction of the cut axonal end. In contrast, our functional measures for seal formation in earthworm MGAs are all consistent with the conclusion that MGAs completely seal within $1 \mathrm{hr}$ after transection in standard salines, that is, $v_{m}$ and $I_{\text {inj }}$ return to values recorded in intact axons and $R_{\text {in }}$ increases above precut values. Our functional measures also suggest that earthworm MGAs do not seal if extracellular calcium is greatly reduced.

\section{Mechanisms of seal formation}

We have evaluated our functional and morphological data from transected GAs and MGAs with particular reference to three sealing mechanisms: (1) sealing by constriction of the cut axonal end followed by fusion of the apposed axolemma, (2) sealing by the appearance of a continuous single membrane across the cut axonal end, or (3) sealing by large injury-induced vesicles that plug the cut axonal end and possibly fuse with each other (see Fig. 9).

(1) Axonal constriction followed by axolemmal fusion as a possible sealing mechanism. The most commonly proposed mechanism for sealing (Fig. $9 A$ ) is constriction of the cut axonal end followed by fusion of the constricted axolemmal membranes (Kao et al., 1977, 1983; Kelley, 1985; Meiri et al., 1987; Spira et al., 1993). However, no study has presented compelling electrophysiological or morphological evidence for complete closure of the opening at the cut end by axolemmal constriction, or evidence for fusion of constricted axolemmal membranes at the cut end. For example, although Kao et al. (1977, 1983) reported that dog axons sealed by a constriction/fusion mechanism, no functional measures of sealing were made and the transected axons were so small and convoluted that the orientation of the end of individual axons could not be confirmed in serial sections. Gallant (1988) has reported 95\% (but not 100\%) constriction of transected squid GAs viewed with dark-field microscopy. Others reporting this mechanism of sealing in severed cockroach axons (Meiri et al., 1987) or severed Aplysia axons (Spira et al., 1993) present no photographic evidence of complete axonal constriction, much less a continuous membrane at the cut end.

We find that squid GAs and earthworm MGAs transected in standard salines do constrict, but the constriction does not completely close the opening at the cut ends. In fact, squid GAs constrict to a greater extent $(\sim 90 \%)$ at their cut axonal ends than earthworm MGAs $(\sim 60 \%)$-and our functional data suggest that squid GAs do not seal within $2.5 \mathrm{hr}$, whereas earthworm MGAs completely seal within $1 \mathrm{hr}$. Furthermore, tight

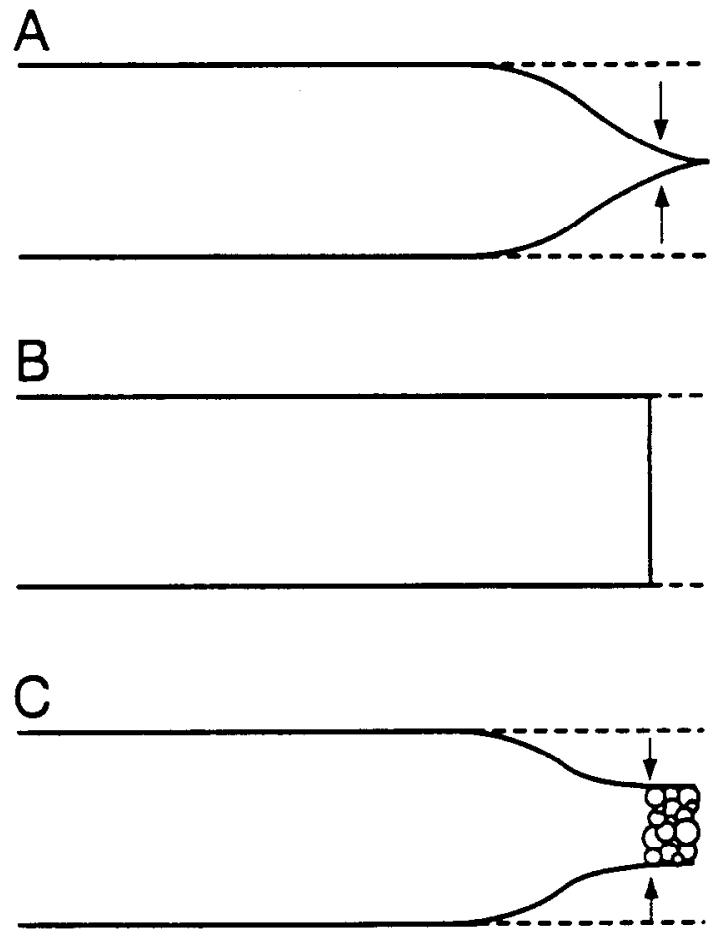

Figure 9. Schematic diagrams of several possible mechanisms responsible for axonal sealing. Axolemmal membranes of transected axons are represented by solid lines, whereas seal membranes are represented by dashed lines. $A$, Sealing by constriction of the cut axonal end followed by fusion of the apposed axolemma. $B$, Sealing by the appearance of a continuous single membrane spanning the opening at or near the cut axonal end. $C$, Sealing mediated by large injury-induced vesicles that plug the cut axonal end, which may be partially constricted.

apposition of membranes at the cut end was never seen in any transected GA or MGA. Spira et al. (1993) have suggested that most of the recovery in $R_{\text {in }}$ takes place as the diameter of a cut axonal end constricts from $0.01 \mu \mathrm{m}$ to closure. If this argument were applied to our preparations, then $R_{\text {in }}$ recovery measured in squid GAs and earthworm MGAs should be associated with constriction of the cut axonal ends to a fraction of a micron. However, we find that when $R_{\text {in }}$ reaches its maximal value in transected GAs and MGAs, a large $(20-70 \mu \mathrm{m})$ opening (filled with vesicles) always remains at the cut axonal ends. Since this large opening exists at the cut end of earthworm MGAs at a time when this axon has completely sealed according to our functional measures, these data strongly suggest that MGAs do not seal by axonal constriction followed by axolemmal fusion.

(2) Appearance of new membrane as a possible sealing mechanism. Yawo and Kuno (1985) have suggested that a "partitionlike structure" (Fig. 9B) might seal off cockroach axons about $150 \mu \mathrm{m}$ from the cut end viewed with phase-contrast microscopy. The spontaneous formation of such a membrane across a gap of tens of micrometers (the diameter of the open axonal ends) should be accompanied by a rapid ( $<1 \mathrm{sec}$ ), dramatic, increase in $R_{\text {in }}$ and decrease in $I_{\text {inj. }}$. We find no such $R_{\text {in }}$ or $I_{\text {inj }}$ changes in transected squid GAs or earthworm MGAs. Further, we find no evidence of a partition-like structure within 4000 $\mu \mathrm{m}$ of the cut ends of squid GAs and earthworm MGAs transected in standard salines viewed with phase-contrast, VE-DIC, or electron microscopy.

(3) Injury-induced vesicles as a possible sealing mechanism. Injury-induced vesicles have long been known to exist (Ramon y Cajal, 1928; Levi and Meyer, 1945) and accumulate at sites 
of axonal damage (Zelena et al., 1968; Kao et al., 1977; Bird, 1978; Smith, 1980; Emery et al., 1987; Meller, 1987) in vertebrate axons. Our data from both living and fixed preparations show that injury-induced vesicles form and accumulate at the cut ends of squid GAs and earthworm MGAs transected in standard salines. These findings are consistent with earlier reports of injury-induced vesiculation in living squid GAs (Fishman et al., 1990; Gallant, 1992) and in living and/or fixed axons from frog sciatic (Lubinska, 1956), rabbit sciatic (Blumcke et al., 1966), rat sciatic (Zelena et al., 1968), dog spinal cord (Kao et al., 1977), chick dorsal root ganglia (Bird, 1978), and mouse spinal neurons in culture (Lucas et al., 1985). We occasionally observed extremely largc vesicles $(\geq 50 \mu \mathrm{m})$ protruding from cut ends of GAs and MGAs in living preparations. It is unlikely that the large vesicles seen in earthworm MGAs arise from septal membranes as reported by Brink and Fan (1989), since such vesicles are localized to the cut axonal end that are hundreds of micrometers removed from the septa and since similar vesicles also protrude from the cut ends of squid GA (Fig. 4G) and frog sciatic axon (Lubinska, 1956), which lack septa. Our observation of the formation of vesicles within living GAs and MGAs subsequent to transection, and the absence of such vesicles in intact axons (Figs. $5 A, C ; 7 A, C$; Coggeshall, 1965; Villegas and Villegas, 1984) make it unlikely that the vesicles seen in fixed preparations are artifacts of the fixation procedure.

A role for injury-induced vesicles in axonal repair and regeneration has only been recently proposed (Fishman et al., 1990). Our data show that vesicles in squid GAs are loosely suspended in a matrix of cytoskcletal debris at $2.5 \mathrm{hr}$ posttransection. Such loosely packed vesicles might not produce a tight seal. In contrast, vesicles in earthworm MGAs at $1 \mathrm{hr}$ posttransection are densely packed at the cut end. Such tightly packed vesicles might produce a seal. Progressive vesicle packing at the cut ends of MGAs could be responsible for the gradual recovery of $R_{\text {in }}$ and gradual decline of $I_{\text {inj; }}$, that is, a gradual increase in the packing of vesicles would lead to a gradual increase in the resistance of a vesicle plug (Fricke and Morse, 1925). Previous functional studies of axonal sealing (Borgens et al., 1980; Meiri et al., 1981; Yawo and Kuno, 1983, 1985; Spira et al., 1993) have also reported gradual recoveries in their measures of sealing similar to that which we report for the earthworm MGA.

\section{Conclusions from our functional and morphological measures of sealing}

Data from our clectrophysiological and morphological measures of earthworm MGAs within $1 \mathrm{hr}$ posttransection are not consistent with sealing by constriction (Fig. $9 A$ ) or the formation of "partition-like structures" (Fig. $9 B$ ). In contrast, all our data from electrophysiological and morphological measures of the MGA are consistent with sealing by the formation of a vesicular plug (Fig. 9C). The incomplete functional recovery of the squid GA is also associated with the accumulation of vesicles at the cut end. The difference between the ineffective sealing of the squid GA and the effective sealing of the earthworm MGA may be that injury induced vesicles are loosely packed at the cut end of the GA and tightly packed in the MGA. While diameter cannot be ruled out as a factor in vesicular sealing, transected squid GAs constrict to a diameter that equals that of constricted earthworm MGAs. Future studies of squid GAs, earthworm MGAs, and other axons should provide insight into the parameters (vesicle size, axon diameter, vesicle fusions, glial wrapping, etc.) necessary to form a tightly packed vesicular plug.
We suggest that many invertebrate and vertebrate axons rapidly form a vesicular plug to produce a functional seal at cut axonal ends as the necessary first step for axonal survival and regeneration. In fact, in other morphological studies we have noted a vesicular plug forms at or near the cut ends of unmyelinated MGAs of crayfish giant axons and myelinated axons in frog and rat peripheral nerves. For any such axon to survive transection, this vesicular plug must effectively separate the intracellular from the extracellular fluid. Ion channels (Fishman et al., 1990) and ion pumps in injury-induced vesicles at or near the cut end may help regulate ionic concentrations in the axon shortly after its transection. In regenerating axons, this vesicular plug is prcsumably replaced by a growth cone at the cut end. Since submission of this article for publication, Steinhardt et al. (1994) reported that vesicles are involved in the sealing of sea urchin embryos and 3 T3 fibroblasts following membrane puncture with a microelectrode. We suggest that the formation of a vesicular plug to repair large or small breaks in the plasmalemma may be a basic cellular mechanism common to many cell types in many phyla.

\section{References}

Bird M (1978) Microsurgical transection of small nerve bundles in vitro: effects on axons, growth cones and glial cells. Cell Tissue Res 190:525-538.

Birse SC, Bittner GD (1981) Regeneration of earthworm giant axons following transection or ablation. J Neurophysiol 45:724-741.

Bittner GD (1991) Long term survival of anucleate axons and its implications for nerve regeneration. Trends Neurosci 14:188-193.

Blumcke S, Niedorf HR, Rode J (1966) Axoplasmic alterations in the proximal and distal stumps of transected nerves. Acta Neuropathol (Berl) 7:44-61.

Borgens R, Jaffe L, Cohen H (1980) Large and persistent electrical currents enter the transected lamprey spinal cord. Proc Natl Acad Sci USA 77:1209-1213.

Borgens RB, Robinson KR, Vanable JW, McGinnis ME (1989) Electric fields in vertebrate repair: natural and applied voltages in vertebrate regeneration and healing. New York: Liss.

Brink PR, Barr L (1977) The resistance of the septum of the median giant axon of the earthworm. J Gen Physiol 69:517-536.

Brink PR, Fan SF (1989) Patch clamp recordings from membranes which contain gap junction channels. Biophys J 56:579-593.

Brown A, Lasek RJ (1990) The cytoskeleton of the squid giant axon. In: Squid as experimental animals (Gilbert DL, Adelman WJ, Arnold JM, eds), pp 235-302. New York: Plenum.

Bullock TH, Horridge GA (1965) Structure and function in the nervous systems of invertebrates. San Francisco: Freeman.

Coggeshall RE (1965) A fine structural analysis of the ventral nerve cord and associated sheath of Lumbricus terrestris L. J Comp Neurol $125: 393-438$.

Délèze J (1970) The recovery of resting potential and input resistance in sheep heart injured by knife or laser. J Physiol (Lond) 208:547562.

De Mello W (1973) Membrane sealing in frog skeletal muscle fibers. Proc Natl Acad Sci USA 70:982-984.

Emery DG, Lucas JH, Gross GW (1987) The sequence of ultrastructural changes in cultured neurons after dendrite transection. Exp Brain Res 67:41-51.

Fishman HM, Tewari KP, Stein PG (1990) Injury-induced vesiculation and membrane redistribution in squid giant axon. Biochim Biophys Acta 1023:421-435.

Gallant PE (1988) Effects of the external ions and metabolic poisoning on the constriction of the squid giant axon after axotomy. J Neurosci 8:1479-1484.

Gallant PE (1992) The direct effects of graded axonal compression on axoplasm and fast axoplasmic transport. J Neuropathol Exp Neurol $51: 220-230$.

George EB, Schneider BF, Lasek RJ, Katz MJ (1988) Axonal shortening and mechanisms of axonal motility. Cell Motil Cytoskel 9:4859. 
Gilbert DL, Adelman WJ, Arnold JM (1990) Squid as experimental animals. New York: Plenum.

Günther J (1976) Impulse conduction in the myelinated giant fibers of the earthworm. Structure and function of the dorsal nodes in the medial giant fiber. J Comp Neurol 168:505-532.

Hodgkin AL, Keynes RD (1957) Movements of labelled calcium in squid giant axons. J Physiol (Lond) 138:253-281.

Jack JJB, Noble D, Tsien RW (1983) Electrical current flow in excitable cells. Oxford: Clarendon.

Jaffe LF, Nuccitelli R (1974) An ultrasensitive vibrating probe for measuring steady extracellular currents. J Cell Biol 63:614-628.

Kao CC, Chang LW, Bloodworth JMB (1977) Electron microscopic observation of the mechanism of terminal club formation in transected spinal cord axons. J Neuropathol Exp Neurol 36:140-156.

Kao CC, Wrathall JR, Kyoshimov K (1983) Axonal reaction to transection. In: Spinal cord reconstruction (Kao CC, Bunge RP, Reier PJ, eds), pp 41-57. New York: Raven.

Kelly JP (1985) Reaction of neurons to injury. In: Principles of neural science (Kandel E, Schwartz J eds), p 188. Amsterdam: Elsevier/North Holland.

Krause TL, Bittner GD (1990) Rapid morphological fusion of severed myelinated axons by polyethylene glycol. Proc Natl Acad Sci USA 87:1471-1475.

Lee PG, Turk PE, Yang WT, Hanlon RT (1994) Biological characteristics and biomedical applications of the squid Sepioteuthis lessoniana cultured through multiple generations. Biol Bull, in press.

Leech CA, Treherne JE (1984) Growth and ion-specificity of excitability in regenerating cockroach giant interneurons. J Exp Biol 110: 311-318.

Levi G, Meyer H (1945) Reactive, regressive, and regenerative processes of neurons, cultivated in vitro and injured with the micromanipulator. J Exp Zool 99:141-181.

Lubinska L (1956) Outflow from cut ends of nerve fibers. Exp Cell Res 10:40-47.

Lucas JH (1987) Proximal segment retraction increases the probability of nerve cell survival after dendrite transection. Brain Res 425:384387.

Lucas JH, Gross GW, Emery DG, Gardner CR (1985) Neuronal survival or death after dendrite transection close to the perikaryon: correlation with electrophysiologic, morphologic and ultrastructural changes. Cent Nerv Syst Trauma 2:231-255.

Lyckman AW, Heidelbaugh SM, Bittner GD (1992) Analysis of neuritic outgrowth from severed giant axons in Lumbricus terrestris. J Comp Neurol 317:1-13.

Meiri H, Spira M, Parnas I (1981) Membrane conductance and action potential of a regenerating axon tip. Science 211:709-712.
Meller K (1987) Early structural changes in the axoplasmic cytoskeleton after axotomy studied by cryofixation. Cell Tissue Res 250:663672.

Nishiye $\mathrm{H}$ (1977) The mechanism of $\mathrm{Ca}^{2+}$ action on the healing-over process in mammalian cardiac muscles: a kinetic analysis. Jpn J Physiol 27:451-466.

Ramon y Cajal S (1928) Degeneration and regeneration of the nervous system (May RM, tráns). London: Oxford UP.

Scheffey C (1988) Two approaches to construction of vibrating probes for electrical current measurement in solution. Rev Sci Instrum 5:787792.

Sereni E, Young JZ (1932) Nervous degeneration and regeneration in cephalopods. Stazione Zool Napoli 12:173-208.

Smith PJS, Shipley A (1990) Regional variation in the current flow across an insect blood-brain barrier. J Exp Biol 154:371-382.

Smith RS (1980) The short term accumulation of axonally transported organelles in the region of localized lesions of single myelinated axons. J Neurocytol 9:39-65.

Sole G (1980) The effects of microsurgical transection of neurite bundles in vitro before and after exposure to colchicine. J Anat 130:777788.

Spira ME, Banbassat D, Dormann A (1993) Resealing of the proximal and distal cut ends of transected axons: electrophysiological and structural analysis. I Neurobiol 24:300-316.

Steinhardt RA, Guoqiang B, Alderton JM (1994) Cell membrane resealing by a vesicular mechanism similar to neurotransmitter release. Science 263:390-393.

Strautman AF, Cork RJ, Robinson KR (1990) The distribution of free calcium in transected spinal axons and its modulation by applied electrical fields. J Neurosci 10:3564-3575.

Verselis V, Brink PR (1984) Voltage clamp of the earthworm septum. Biophys J 45:147-150.

Villegas GM, Villegas R (1984) Squid axon ultrastructure. In: Current topics in membranes and transport, Vol 22 (Baker PF, ed), pp 3-37. London: Academic.

Weidmann S (1952) The electrical constants of Purkinje fibres. J Physiol (Lond) 118:348-360.

Yawo H, Kuno M (1983) How a nerve fiber repairs its cut end: involvement of phospholipase $A_{2}$. Science 222:1351-1353.

Yawo H, Kuno M (1985) Calcium dependence of membrane sealing at the cut end of the cockroach giant axon. J Neurosci 5:1626-1632.

Zelena J, Lubinska L, Gutman E (1968) Accumulation of organelles at the ends of interrupted axons. Z Zellforsch Microsk Anat 91:200 219 . 